\title{
Antibody-protein interactions: benchmark datasets and prediction tools evaluation
}

\author{
Julia V Ponomarenko*1,2 and Philip E Bourne ${ }^{1,2}$
}

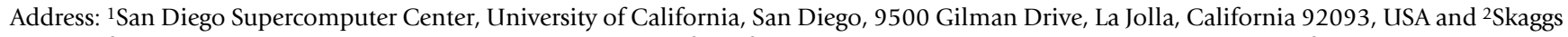
School of Pharmacy and Pharmaceutical Sciences, University of California, San Diego, 9500 Gilman Drive, La Jolla, California 92093, USA

Email: Julia V Ponomarenko* - jpon@sdsc.edu; Philip E Bourne - bourne@sdsc.edu

* Corresponding author

Published: 2 October 2007

BMC Structural Biology 2007, 7:64 doi:10.1 |86/1472-6807-7-64

This article is available from: http://www.biomedcentral.com/l472-6807/7/64

(C) 2007 Ponomarenko and Bourne.; licensee BioMed Central Ltd.

This is an Open Access article distributed under the terms of the Creative Commons Attribution License (http://creativecommons.org/licenses/by/2.0), which permits unrestricted use, distribution, and reproduction in any medium, provided the original work is properly cited.

\begin{abstract}
Background: The ability to predict antibody binding sites (aka antigenic determinants or B-cell epitopes) for a given protein is a precursor to new vaccine design and diagnostics. Among the various methods of B-cell epitope identification X-ray crystallography is one of the most reliable methods. Using these experimental data computational methods exist for B-cell epitope prediction. As the number of structures of antibody-protein complexes grows, further interest in prediction methods using 3D structure is anticipated. This work aims to establish a benchmark for 3D structure-based epitope prediction methods.

Results: Two B-cell epitope benchmark datasets inferred from the 3D structures of antibodyprotein complexes were defined. The first is a dataset of 62 representative 3D structures of protein antigens with inferred structural epitopes. The second is a dataset of 82 structures of antibodyprotein complexes containing different structural epitopes. Using these datasets, eight web-servers developed for antibody and protein binding sites prediction have been evaluated. In no method did performance exceed a $40 \%$ precision and $46 \%$ recall. The values of the area under the receiver operating characteristic curve for the evaluated methods were about 0.6 for ConSurf, DiscoTope, and PPI-PRED methods and above 0.65 but not exceeding 0.70 for protein-protein docking methods when the best of the top ten models for the bound docking were considered; the remaining methods performed close to random. The benchmark datasets are included as a supplement to this paper.

Conclusion: It may be possible to improve epitope prediction methods through training on datasets which include only immune epitopes and through utilizing more features characterizing epitopes, for example, the evolutionary conservation score. Notwithstanding, overall poor performance may reflect the generality of antigenicity and hence the inability to decipher B-cell epitopes as an intrinsic feature of the protein. It is an open question as to whether ultimately discriminatory features can be found.
\end{abstract}

\section{Background}

A B-cell epitope is defined as a part of a protein antigen recognized by either a particular antibody molecule or a particular B-cell receptor of the immune system [1]. The main objective of B-cell epitope prediction is to facilitate the design of a short peptide or other molecule that can be 
synthesized and used instead of the antigen, which in the case of a pathogenic virus or bacteria, may be harmful to a researcher or experimental animal [2]. A B-cell epitope may be continuous, that is, a short contiguous stretch of amino acid residues, or discontinuous, comprising atoms from distant residues but close in three-dimensional space and on the surface of the protein.

Synthetic peptides mimicking epitopes, as well as antipeptide antibodies, have many applications in the diagnosis of various human diseases [3-7]. Also, the attempts have been made to develop peptide-based synthetic prophylactic vaccines for various infections, as well as therapeutic vaccines for chronic infections and noninfectious diseases, including autoimmune diseases, neurological disorders, allergies, and cancers [8-10]. The immunoinformatics software and databases developed to facilitate vaccine design have previously been reviewed $[11,12]$.

During the last 25 years B-cell epitope prediction methods have focused primarily on continuous epitopes. They were mostly sequence-dependent methods based upon various amino acid properties, such as hydrophilicity [13], solvent accessibility [14], secondary structure [15$18]$, and others. Recently, several methods using machine learning approaches have been introduced that apply hidden Markov models (HMM) [19], artificial neural networks (ANN) [20], support vector machine (SVM) [21], and other techniques $[22,23]$. Recent assessments of continuous epitope prediction methods demonstrate that "single-scale amino acid propensity profiles cannot be used to predict epitope location reliably" [24] and that "the combination of scales and experimentation with several machine learning algorithms showed little improvement over single scale-based methods" [25].

As crystallographic studies of antibody-protein complexes have shown, most B-cell epitopes are discontinuous. In 1984, the first attempts at epitope prediction based on 3D protein structure was made for a few proteins for which continuous epitopes were known [26-28]. Subsequently, Thornton and colleagues [29] proposed a method to locate potential discontinuous epitopes based on a protrusion of protein regions from the protein's globular surface. However, until the first X-ray structure of an antibody-protein complex was solved in 1986 [30], protein structural data were mostly used for prediction of continuous rather than discontinuous epitopes.

In cases where the three-dimensional structure of the protein or its homologue is known, a discontinuous epitope can be derived from functional assays by mapping onto the protein structure residues involved in antibody recognition [31]. However, an epitope identified using an immunoassay may be an artefact of measuring cross-reac- tivity of antibodies due to the presence of denatured or degraded proteins $[32,33]$, or due to conformational changes in the protein caused by residue substitutions that may even lead to protein mis-folding [34]. Therefore, structural methods, particularly X-ray crystallography of antibody-antigen complexes, generally identify B-cell epitopes more reliably than functional assays [35].

B-cell epitopes can be thought of in a structural and functional sense. Structural epitopes (also called antigenic determinants) are defined by a set of residues or atoms in the protein antigen contacting antibody residues or atoms $[33,36]$. In contrast, a functional epitope consists of antigen residues that contribute significantly to antibody binding [36,37]. Functional epitopes are determined through functional assays (e.g., alanine scanning mutagenesis) or calculated theoretically using known structures of antibody-protein complexes [38,39]. Thus, functional and structural epitopes are not necessary the same. Functional epitopes in proteins are usually smaller than structural epitopes; only three to five residues of the structural epitope contribute significantly to the antibodyantigen binding energy [40]. This work focuses on structural epitopes inferred from known 3D structures of antibody-protein complexes available in the Protein Data Bank (PDB) [41].

Antibody-protein complexes can be categorized as intermediate transient non-obligate protein-protein complexes $[40,42]$. Non-obligate complexes, implying that individual components can be found on their own in vivo, are classified as either permanent or transient depending on their stability under particular physiological and environmental conditions [43]. For example, many enzymeinhibitor complexes are permanent non-obligate complexes. Transient non-obligate complexes range from weak (e.g., electron transport complexes), to intermediate (e.g., signal transduction complexes), and to strong (e.g., bovine $\mathrm{G}$ protein forming a stable trimer upon GDP binding) [44]. Most antibodies demonstrate intermediate affinity for their specific antigens [45]. Based on this classification, general methods for the prediction of intermediate transient non-obligate protein-protein interactions have been applied to the prediction of structural epitopes $[40,42]$. For example, Jones and Thornton, using their method for predicting protein-protein binding sites [46], successfully predicted B-cell epitopes on the surface of the $\beta$-subunit of human chorionic gonadotropin ( $\beta$ hCG) [47].

Since the number of available structures of antibody-protein complexes remains limited, thus far only a few methods, CEP (Conformational Epitope Prediction) [48] and DiscoTope [49], for B-cell epitope prediction using a protein of a given three-dimensional structure have been 
developed. In the near future, with growth in the number of available structures of antibody-protein complexes, extensive development in this area is expected. Existing and new methods for epitope prediction demand a benchmark which will set the standard for the future comparison of methods. To facilitate the further development of this standard, we have developed B-cell epitope benchmark datasets inferred from existing 3D structures of antibody-protein complexes. Further, using the benchmark datasets, we evaluated CEP, DiscoTope, and six recently developed publicly available web-servers for generalized protein-protein binding site prediction using various approaches: protein-protein docking (ClusPro [50], DOT [51] and PatchDock [52]); structure-based methods applying different principals and trained on different datasets (PPI-PRED [53], PIER [54] and ProMate [55]), and residue conservation (ConSurf [56]).

\section{Results and discussion Structural epitope definition}

Three definitions of an epitope inferred from the X-ray structures of antibody-protein complexes were considered: (1) The epitope consists of protein antigen residues in which any atom of the residue looses more than $1 \AA^{2}$ of accessible surface area (ASA) upon antibody binding. ASA was calculated using the program NACCESS [57]; (2) The epitope consists of protein antigen residues in which any atom of the epitope residue is separated from any antibody atom by a distance $\leq 4 \AA$; (3) The epitope consists of protein antigen residues in which any atom of the epitope residue is separated from any antibody atom by a distance $\leq 5 \AA$. These three definitions were used for two reasons. First, the methods evaluated in this work use one of these three definitions, second, we wished to study how the epitope definition influenced the results.

Results (not shown) indicated that the structural epitope definition did not influence the outcome. Hence, unless otherwise specified, results are based on the second epitope definition.

\section{Construction of the benchmark datasets}

Two benchmark datasets were derived from the 3D structures of antibody-protein complexes available from the PDB [41]:

- Dataset \#1 - Representative 3D structures of protein antigens with structural epitopes inferred from 3D structures of antibody-protein complexes. This dataset is intended for the study of the antigenic properties of proteins as well as for development and evaluation of the methods based on protein structure alone, or protein-protein unbound docking methods, that is, if the structure of the antibody is known or can be modeled. Here this dataset was used for the evaluation of scale-based methods
(DiscoTope, PIER, ProMate and ConSurf). The dataset contains 62 antigens, 52 of which are one-chain antigen proteins.

- Dataset \#2 - Representative 3D structures of antibodyprotein complexes presenting different epitopes. This dataset is useful for the study of the properties of individual epitopes as well as for development and evaluation of protein-protein bound docking methods. Since the current work attempts to compare the methods of different types, including protein-protein docking methods, this dataset was used to compare the performance of all methods to each other. The dataset contains 70 structures of proteins in complexes with two-chain antibodies and 12 structures of proteins in complexes with one-chain antibodies.

The flowchart describing the construction of the benchmark datasets is shown in Figure 1. Steps from 1 to 4 relate to dataset \#1; steps 1-6 relate to dataset \#2.

Step 1 - crystal structures of protein antigens of length $\geq 30$ amino acids at a resolution $\leq 4 \AA$ in complex with antibody fragments containing variable regions (Fab, VHH, $\mathrm{Fv}$, or scFv fragments) were collected from the Protein Data Bank (PDB) [41]. Structures in which the antibody binds antigen but involves no CDR residues have been excluded from the analysis; there were four such structures [PDB: 1MHH, 1HEZ, 1DEE, 1IGC]. If a structure contained several complexes in one asymmetric unit and there was no structural difference observed between these complexes, only one complex was selected. In this way 166 structures containing 187 antibody-protein complexes were selected: 24 complexes were formed by onechain antibody fragments and 163 complexes by twochain antibody fragments.

Step 2 - all antigen protein chains were structurally aligned to one another using the CE algorithm [58]. Two protein chains were considered similar if all the following conditions applied: (i) rmsd $\leq 3 \AA$, (ii) z-score $\geq 4.0$, (iii) number of residue-residue matches relative to the length of the longest chain $\geq 80 \%$, (iv) sequence identity in the structural alignment (not considering gaps) $\geq 80 \%$. The zscore takes into account overall structural similarity and number of gapped positions. Two protein molecules were considered similar if each chain in one protein had a similar chain in another protein. Figure 2 demonstrates how the last two parameters, number of matches and sequence identity in the structural alignment, are defined.

The structural alignment rather than sequence alignment was used because protein structure is more conserved than sequence, and there can be expected regions in proteins with low sequence similarity that cannot be aligned by 


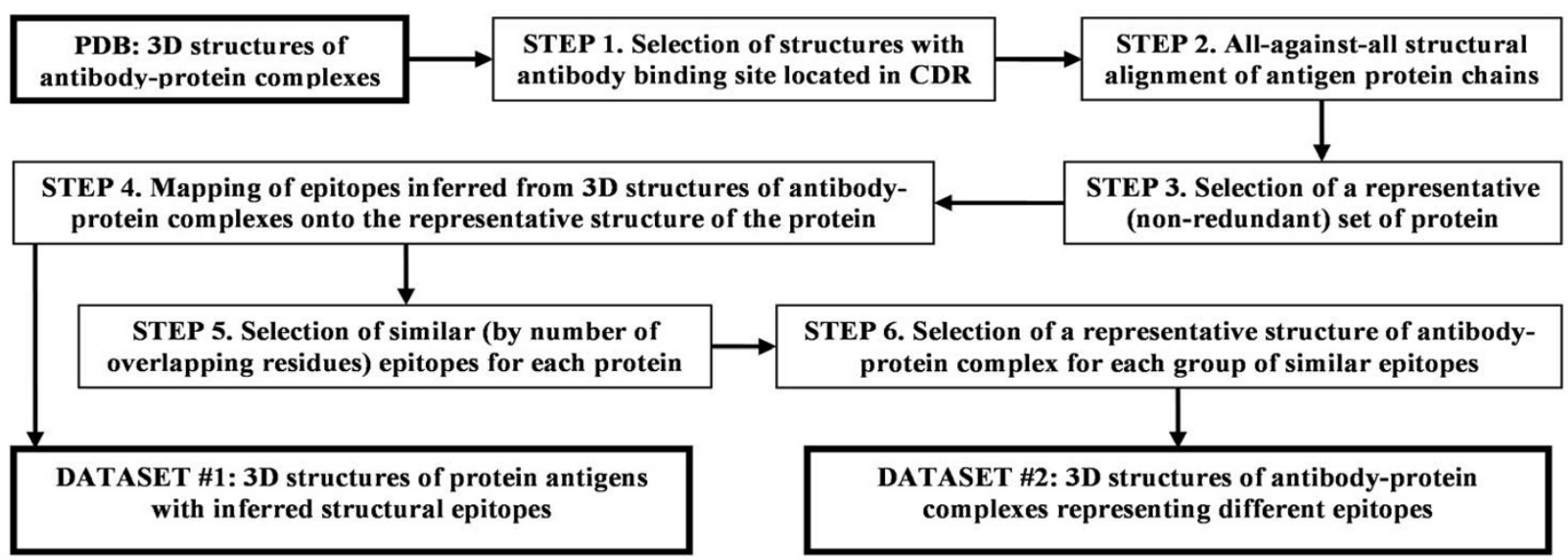

Figure I

Flowchart for building benchmark datasets.

sequence alone. The structural alignment also avoids considering two proteins as similar if they have similar sequences but different structures (possible over short regions). The threshold values were chosen empirically based on previous experience working with the CE algorithm. As a result, the chosen threshold values separated human and bird lysozymes (61\% sequence identity) and neuraminidases of different influenza virus strains, H3N2 and H11N9 (47\% sequence identity).

Step 3 - 35 proteins were orphans represented by only one $3 \mathrm{D}$ structure. Of the remaining 27 proteins represented by more than one $3 \mathrm{D}$ structure, the structure with the best resolution was selected as the representative structure. The final representative dataset contained 62 antigens [see Additional file 1], 52 of which were one-chain antigen proteins.

\section{(A) AVCQ---YWC (B) A-CYARTY-C}

Figure 2

Hypothetical example of the structural alignment of proteins (A) (sequence AVCQYWC) and (B)

(sequence ACYARTYC). Number of residue-residue matches $=5$, number of residue-residue matches relative to the length the longest chain $=63 \%(5 / 8)$, sequence identity $=$ $80 \%(4 / 5)$.
Step 4 - for each protein, epitopes inferred from the 3D structures of antibody-protein complexes were mapped onto the representative structure of the protein. First, epitope residues were calculated for each complex structure using one of the aforementioned epitope definitions. Second, epitope residues defined for the represented structures were mapped onto the representative structure based on the structure alignments. For example, the hemagglutinin HA1 chain of influenza A virus was represented by six $3 \mathrm{D}$ structures of the protein in complexes with Fab fragments of antibodies HC45 [PDB:1QFU], BH151 [PDB:1EO8], HC63 [PDB:1KEN], and HC19 [PDB:2VIR, 2VIS, 2VIT]. Figure 3 illustrates a representative structure [PDB:1EO8] of hemagglutinin HA1 upon which epitopes are mapped having been inferred from six complex structures. In this way, epitopes inferred from 187 structures of antibody-protein complexes were mapped onto the 62 representative protein structures. The resulting dataset is denoted dataset \#1. Data on mapped epitope residues are available upon request.

Step 5 - to study the properties of individual epitopes and their prediction a dataset of representative epitopes, dataset \#2 derived from 3D structures of antibody-protein complexes defining different epitopes was constructed. An important question to consider is how to define individual epitopes yet avoid bias by over-presentation of particular epitopes? For example (Fig. 3), while HC45 (blue) and BH151 (magenta) epitopes overlap, neither HC63 (green) nor HC19 (red) epitopes overlap, they are separated on the protein surface. Nevertheless, HC45 and BH151 epitopes share residues (orange in Fig. 3), as do HC63 and HC19 epitopes (yellow in Fig. 3). Are HC45 

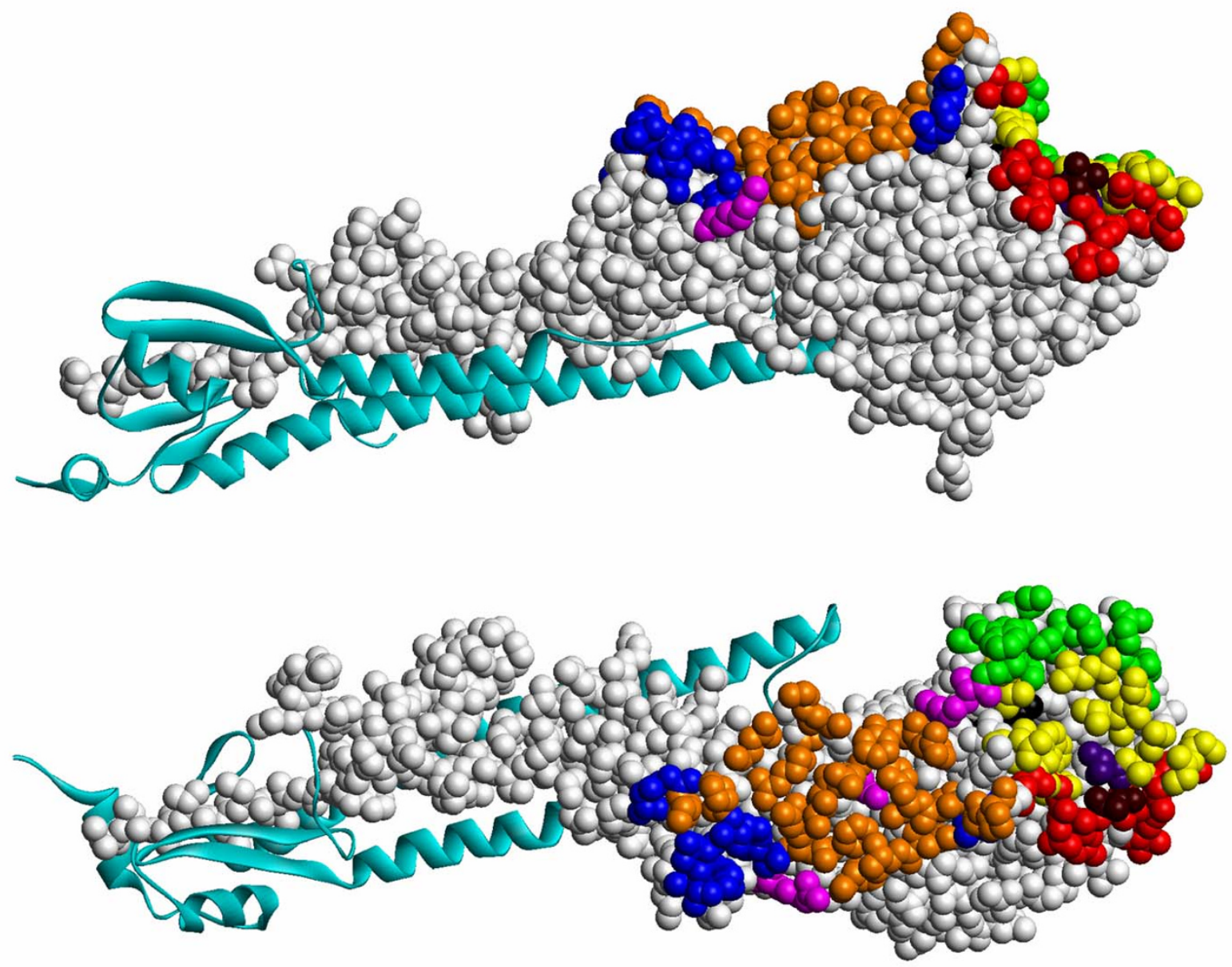

Figure 3

Two orthogonal views of a representative structure, influenza A virus hemagglutinin HAI chain [PDB:IEO8]. Chain $A$ is shown in light gray upon which are mapped epitope residues inferred from six protein structures in complexes with antibody fragments: HC45 Fab [PDB:IQFU] (blue), BHI5I Fab [PDB:IEO8] (magenta), HC63 Fab [PDB:IKEN] (green), HCI9 Fab [PDB:2VIR, 2VIS, 2VIT] (red). The hemagglutinin HA2 chain is shown in cyan. Residues common to HC45 and BHI5I epitopes are shown in orange; residues common to $\mathrm{HC} 63$ and $\mathrm{HCl} 9$ epitopes are shown in yellow; residue Tyr98 which is a part of HCI 9 epitope inferred from structure 2VIR but not from 2VIS and 2VIT structures is shown in black; The HCI9 epitope residue ThrI3I which is mutated to lle in the 2VIS structure is shown in dark red. The HCI9 epitope residue Thr I55 which is mutated to lle in $2 \mathrm{VIT}$ structure is shown in violet.

and BH151 epitopes similar or different? This question is answered by considering the degree of overlap.

Two epitopes are deemed similar if, in addition to the aforementioned criteria for epitope definition, they belong to similar protein chains and have $>75 \%$ residues in common for both epitopes. A cut-off value of $75 \%$ for epitope similarity was chosen empirically. Thus, the HC45 and BH151 epitopes on influenza A virus hemagglutinin HA1 (Fig. 3) share 14 residues, that make up 74\% and $93 \%$ of the size of HC45 and BH151 epitopes, respectively. A cut-off on epitope overlap of less than $75 \%$ would define HC45 and BH151 epitopes as similar even though they are known to be different. HC45 and BH151 are antibodies from different germ-lines with variable domains sharing only $56 \%$ sequence similarity, their $\mathrm{H} 3$ CDR regions adopt distinct conformations and these antibodies are tolerant to different mutations in hemagglutinin [59]. Another example, X5 and 17B epitopes of gp120 share $75 \%$ of their residues yet $\mathrm{X} 5$ and $17 \mathrm{~B}$ antibodies are from different genes [60]. A cut-off value for epitope similarity equal to or less than $75 \%$ would erroneously define $\mathrm{X} 5$ and 17B epitopes as similar. Conversely, a cut-off value of $80 \%$ would make epitopes inferred from different 
structures of the same antibody-protein complex dissimilar. For example, the H57 epitope of T cell receptor N15 is inferred from two complex structures of a single crystal asymmetric unit ([PDB:1NFD], complexes (D)-(HG) and (B)-(FE), where the letters denote protein chain identifiers) would be dissimilar.

Given a 75\% empirical cut-off for epitope similarity, epitopes inferred from structures of complexes with twochain antibody fragments were divided into 44 singletons and 26 groups; epitopes inferred from structures of complexes with one-chain antibody fragments were divided into ten singletons and two groups.

Step 6 - for each group of similar epitopes, the representative 3D structure of the antibody-protein complex was selected based upon the following preferences. First, the structure with no or a minimal number of heteroatoms (excluding water) and other protein chains in the interface (i.e., separated from any atoms of both antigen and antibody by $\leq 4 \AA$ distance) was preferred. Second, preference was given to the structure with the largest epitope, i.e., maximum number of epitope residues. Third, the structure with the best resolution $\leq 2.5 \AA$ was preferred. Dataset $\# 2$ of representative structures of antibody-protein complexes (representative epitopes) consisted of 70 structures of proteins in complexes with two-chain antibody fragments and 12 structures of proteins in complexes with one-chain antibody fragments.

\section{Web-servers performance evaluation}

Using the benchmark datasets introduced above we evaluated eight recently-developed and publicly available web-servers. The servers use different methods yet all have the goal of predicting either B-cell epitopes, or more generally protein-protein binding sites. The servers are listed in Table 1 . Any reference in the text to the method actually means the server which implements that method, e.g., the DOT method running on the ClusPro server is called ClusPro(DOT).

The methods fall into two categories:

- Scale-based methods - each protein residue is assigned a value reflecting the probability of that residue being part of the protein interface or epitope. DiscoTope, PIER, ProMate and ConSurf fall into this category.

- Patch prediction and protein-protein docking methods - each protein residue is predicted to be part of a surface patch of residues defining the protein interface or epitope. DiscoTope, ProMate, CEP, PPI-PRED, ClusPro(DOT), and PatchDock fall into this category.

Two methods, DiscoTope and ProMate, fall into both categories since they predict patches and assign score values to each protein residue.

The evaluation of the methods was performed as follows. First, the scale-based methods were analyzed on how well the residue score values discriminate epitope versus nonepitope residues using dataset \#1. Further, performance of all methods was evaluated on their ability to recognize representative epitopes from dataset \#2. The first step is obviously not essential; it was performed as an example of the application of dataset \#1 that can be used for future

Table I: Servers evaluated in this work

\begin{tabular}{|c|c|c|c|}
\hline Server name & Method type & Training dataset & Reference \\
\hline $\begin{array}{l}\text { CEP (Conformational } \\
\text { Epitope Prediction) }\end{array}$ & $\begin{array}{l}\text { Discontinuous epitope prediction based on residue } \\
\text { solvent accessibility and spatial distribution. }\end{array}$ & No training set. & {$[48]$} \\
\hline DiscoTope & $\begin{array}{l}\text { Discontinuous epitope prediction based on amino } \\
\text { acid statistics, residue solvent accessibility and spatial } \\
\text { distribution. }\end{array}$ & 75 structures of antibody-antigen complexes. & {$[49]$} \\
\hline ProMate & $\begin{array}{l}\text { Protein-protein binding interface prediction based on } \\
\text { significant structural and sequence interface } \\
\text { properties. }\end{array}$ & $\begin{array}{l}\text { Manually curated; } 57 \text { protein involved in } \\
\text { heterodimeric transient interactions (excluding } \\
\text { antigen-antibody complexes). }\end{array}$ & {$[55]$} \\
\hline $\begin{array}{l}\text { PIER (Protein IntErface } \\
\text { Recognition) }\end{array}$ & $\begin{array}{l}\text { Protein-protein binding interface prediction based on } \\
\text { local statistical properties of the protein surface } \\
\text { derived at the level of atomic groups. }\end{array}$ & $\begin{array}{l}490 \text { homodimeric, } 62 \text { heterodimeric and } 196 \\
\text { transient interfaces (excluding antigen-antibody } \\
\text { complexes). }\end{array}$ & {$[54]$} \\
\hline $\begin{array}{l}\text { PPI-PRED (Protein- } \\
\text { Protein Interface } \\
\text { Prediction) }\end{array}$ & $\begin{array}{l}\text { Protein-protein binding interface prediction based on } \\
\text { significant structural and sequence interface } \\
\text { properties. }\end{array}$ & $\begin{array}{l}\text { Manually curated; I } 80 \text { proteins from } 149 \text { complexes } \\
\text { both obligate (I } 14) \text { and transient }(66) .\end{array}$ & {$[53]$} \\
\hline ConSurf & $\begin{array}{l}\text { Mapping of phylogenetic information (sequence } \\
\text { conservation grades) on to the surface of proteins } \\
\text { with known 3D structure. }\end{array}$ & No training set. & {$[56]$} \\
\hline $\begin{array}{l}\text { ClusPro (DOT } \\
\text { program) }\end{array}$ & $\begin{array}{l}\text { Rigid-body protein-protein docking based on the } \\
\text { Fast-Fourier Transform correlation approach. }\end{array}$ & No training set. & {$[50][51]$} \\
\hline PatchDock & $\begin{array}{l}\text { Rigid-body protein-protein docking based on local } \\
\text { shape feature matching. }\end{array}$ & No training set. & {$[52]$} \\
\hline
\end{tabular}


methods development and for revealing properties of epitope residues beyond the fact that epitopes are sites on the protein surface.

Scale-based methods: score value distributions

DiscoTope, PIER, ProMate and ConSurf assign to each protein residue a score reflecting the probability of that residue being a part of the protein interface or epitope. Details are provided in the Methods section. For the analysis of epitope residues versus non-epitope residues we used dataset \#1, that is, representative antigen structures with epitopes mapped onto them. Here an epitope residue is an antigen residue known to be part of an epitope in any complex of this antigen with any antibody. Conversely a non-epitope residue implies an antigen residue which is not known to be part of a structural epitope. To simplify the calculation proteins with epitopes located on more than one protein chain were discarded from the analyses (there were 10 such proteins). As a result 52 protein antigens were analyzed [see Additional file 1].

The score distributions for epitope, non-epitope and all protein residues were calculated for each method and are shown in Figures 4, 5, 6, 7. Distributions taking into account only surface residues were similar for all methods (results not shown). The definition of a surface residue is given in the Methods section.

DiscoTope, ProMate and ConSurf scores discriminate epitope versus non-epitope and versus all protein residues, while PIER and ConSurf confidence scores do not. Thus, as one can see in Figure 4, DiscoTope discriminates epitope residues $(\bar{x}=-10.2, \mathrm{~s}=5.4$, number of residues $\mathrm{N}$ $=1,364)$ from non-epitope residues $(\bar{x}=-13.3, \mathrm{~s}=6.3, \mathrm{~N}$ $=9,713)(\mathrm{p}<0.001)$ and all antigen residues $(\bar{x}=-13.0$, $\mathrm{s}=6.3, \mathrm{~N}=11,077)(\mathrm{p}<0.001)$. These distributions are

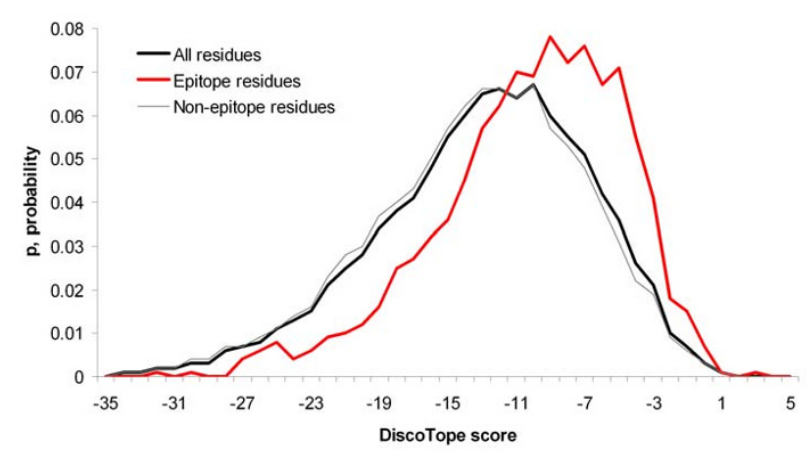

\section{Figure 4}

Distributions of DiscoTope scores for epitope, non-epitope and all protein residues.

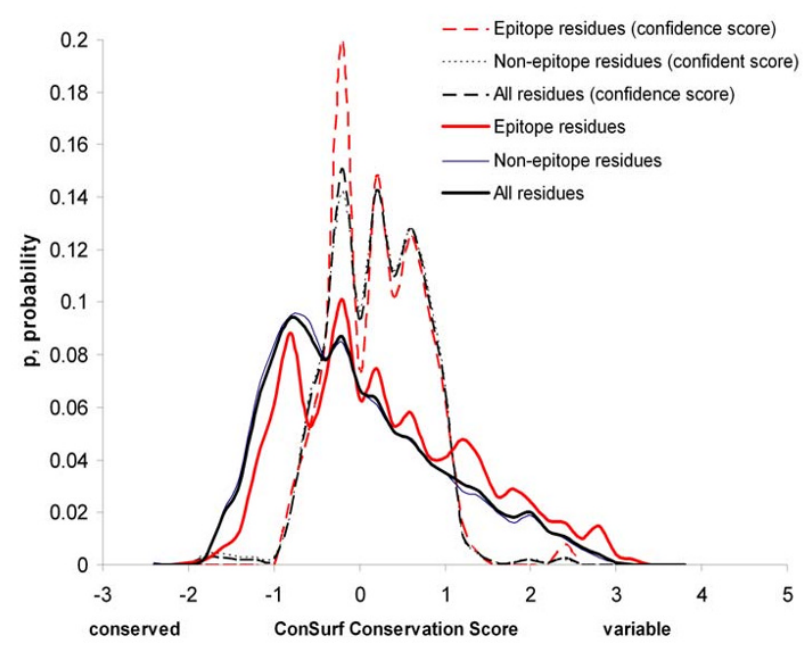

Figure 5

Distribution of ConSurf scores for epitope and all protein residues. For the definition of confidence score see the Methods section.

significantly different $(\mathrm{p}<0.001)$ regardless of the epitope definition used. The ConSurf conservation score also discriminates epitope residues $(\bar{x}=0.273, \mathrm{~s}=1.050, \mathrm{~N}=$ $1,119)$ versus non-epitope residues $(\bar{x}=-0.049, \mathrm{~s}=$ $0.987, \mathrm{p}<0.001)$ and versus all antigen residues $(\bar{x}=$ 0.007, $\mathrm{s}=1.00, \mathrm{~N}=8,684, \mathrm{p}<0.001$ ) (Fig. 5). The same was true for epitope vs. all surface residues. Further, the confidence level did not change when the definition of surface residues and/or epitope residues was changed (data not shown). However, if only residues with ConSurf

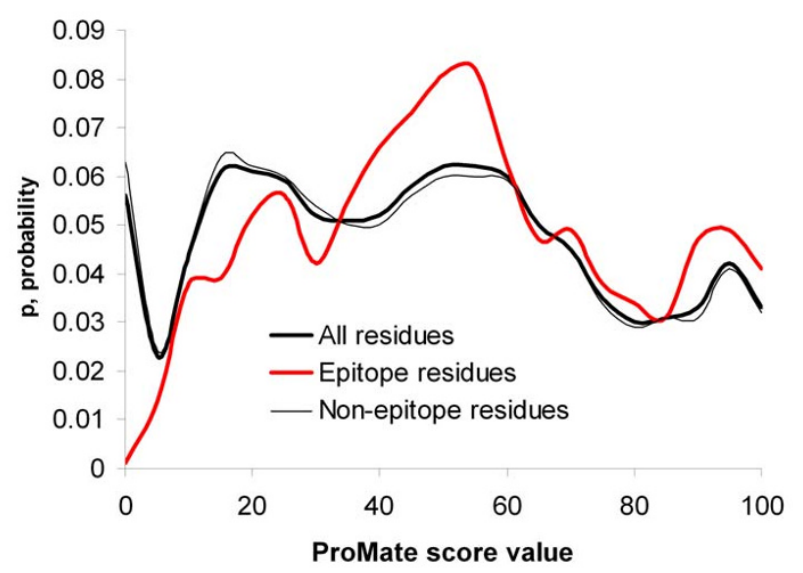

\section{Figure 6}

Distribution of ProMate scores for epitope, non-epitope and all protein residues. 


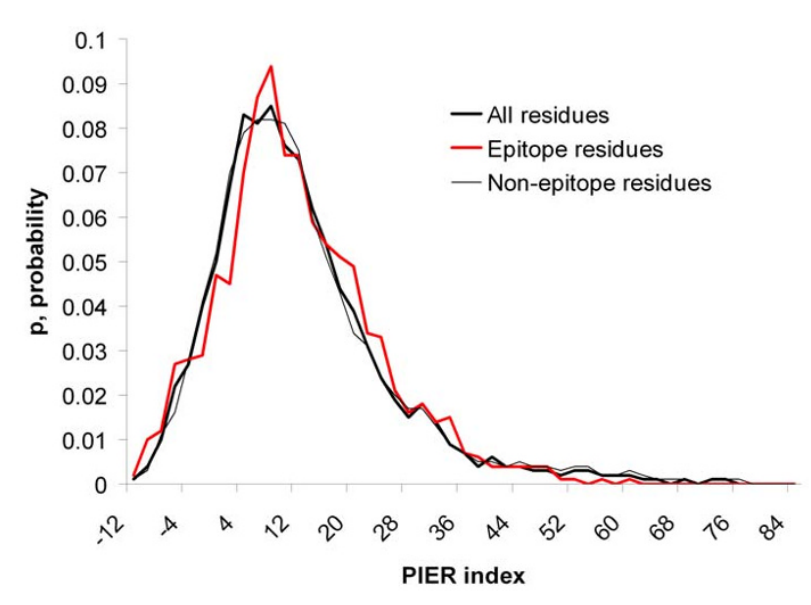

\section{Figure 7}

Distribution of PIER scores for epitope, non-epitope and all protein residues.

confidence score values were considered, no significant difference between epitope and other protein residues was observed (epitope residues: $\bar{x}=0.197, \mathrm{~s}=0.539$; nonepitope residues: $\bar{x}=0.194, \mathrm{~s}=0.556, \mathrm{p}>0.05)$. For ProMate mean scores for epitope residues $(\bar{x}=52.8, \mathrm{~s}=25.4$, $\mathrm{N}=1,363)$ were significantly higher than for all antigen residues $(\bar{x}=46.5, \mathrm{~s}=28.1, \mathrm{~N}=11,074)$ or non-epitope residues or all surface residues $(\mathrm{p}<0.001)$ (Fig. 6). The PIER score does not discriminate epitope versus other antigen residues (epitope residues: $\bar{x}=11.9, \mathrm{~s}=11.4, \mathrm{~N}=$ 1,363; non-epitope residues: $\bar{x}=12.6, \mathrm{~s}=13.7 ; \mathrm{N}=$ 8,221, p > 0.05) (Fig. 7).

These results suggest that epitope residues are less conservative according to the ConSurf evolutionary conservancy scores than protein surface residues in general at a $99.9 \%$ confidence level $(p<0.001)$. PIER, which is trained on 3D structures of all protein-protein complexes available in the PDB, could not distinguish epitopes from the rest of the protein surface. One possible explanation of this failure is that epitope residues do share some properties with residues having transient non-obligate heterointeractions with other proteins. ProMate is trained using such complexes [55].

\section{Criteria and dataset used in methods evaluation}

There is no commonly acceptable standard for evaluating binding site prediction methods. Some authors measure performance on a per protein bases, measuring statistics across the dataset [49], while others measure performance on a per residue basis [54]. Some authors report sensitivity and specificity and measure the performance from the area under the ROC curve [49], while others consider only the sensitivity and positive predictive values and measure the method performance from the relative number of successful predictions in the test dataset [53].

Approaching the task of evaluation and comparison of different methods, we encountered a number of questions. How can we compare scale-based methods with patch prediction and docking methods? DiscoTope and ProMate predict one patch per protein, while other methods predict several patches, how can these be compared? Using a score value assigned by ProMate, DiscoTope, or ConSurf to a residue, all epitopes in the protein are taken into account, so can we say that the method predicts one epitope per protein? Is not the direct comparison of protein docking methods (ClusPro (DOT), PatchDock) versus patch-based prediction methods (DiscoTope, ProMate, CEP, PPI-PRED) questionable since the former methods are based on optimization of an interaction energy function, while the latter depend on training? Finally, docking methods require knowledge of the structures of both interacting proteins, antigen and antibody, while binding site prediction methods are based on the structure of the protein antigen alone and do not require knowledge of the antibody structure. Is this a fair comparison? Being aware of these questions and limitations, we applied various evaluation criteria in an attempt to provide a thorough and fair comparison of the methods.

The evaluation was performed on the dataset of representative epitopes, assuming any antigen residue which is not a part of a considered epitope is part of a non-epitope. We didn't discard non-epitope residues, which we know belong to some other epitope in the protein, because we assumed that a prediction program will predict an epitope in an antigen for which it doesn't have any additional information except its sequence and structure - this is how all evaluated methods were constructed. The analysis was performed using the representative epitopes from dataset \#2 that were inferred from structures of one-chain (monomer) antigens in complexes with two-chain antibody fragments. There were 59 such epitopes in 48 antigens (Table 2).

The following parameters were used to evaluate the methods:

Sensitivity (recall or true positive rate $(T P R))=T P /(T P+$ $F N)$ - a proportion of correctly predicted epitope residues (TP) with respect to the total number of epitope residues $(\mathrm{TP}+\mathrm{FN})$.

Specificity (or $1-$ false positive rate $(F P R))=1-F P /(T N+$ FP) - a proportion of correctly predicted non-epitope residues (TN) with respect to the total number of nonepitope residues $(\mathrm{TN}+\mathrm{FP})$. 


\begin{tabular}{|c|c|c|c|c|c|c|c|c|c|c|c|c|c|c|c|c|c|c|c|c|c|c|c|c|c|}
\hline \multirow[b]{2}{*}{ antigen } & \multirow[b]{2}{*}{ epitope } & \multirow[b]{2}{*}{ antigen size } & \multirow[b]{2}{*}{ epitope size } & \multicolumn{2}{|c|}{ ProMate } & \multicolumn{2}{|c|}{$\begin{array}{l}\text { PPI-PRED } \\
\text { (1st patch) }\end{array}$} & $\begin{array}{r}\text { PPI-PR } \\
\text { (best pa }\end{array}$ & & PatchDock & model & PatchDo & k best mode & of 10 & ClusPro (DO & model & ClusPro & TT) best mo & 1 of 10 & & CEP & & & coTop & $(-7.7)$ \\
\hline & & & & sensitivity & $\mathrm{ppv}$ & sensitivity & $\mathrm{ppv}$ & sensitivity & ppv & sensitivity & ppv & Model \# & sensitivity & $\mathrm{ppv}$ & sensitivity & ppv & Model\# & sensitivity & ppv & $N$ predictions & sensitivity & ppv & Sensitivity & ppr & $\begin{array}{l}\text { Is in } \\
\text { training set:\& }\end{array}$ \\
\hline 2ADF:A & 2adf_A_HL & 196 & 15 & 0 & 0 & 0.8 & 0.67 & 0.8 & 0.67 & 0 & 0 & 4 & 0.4 & 0.29 & 0.67 & 0.5 & 1 & 0.67 & 0.5 & 7 & 0.27 & 0.14 & 0.07 & 0.11 & . \\
\hline 2ADF:A & Ife8_B_IM & 196 & 20 & 0 & 0 & 0.3 & 0.33 & 0.3 & 0.33 & 0 & 0 & 2 & 0.4 & 0.38 & 0.63 & 0.57 & i & 0.63 & 0.57 & 7 & 0.32 & 0.22 & 0.15 & 0.33 & $*$ \\
\hline IAFV:A & lafv_A_HL & 151 & 14 & 0 & 0 & 0.57 & 0.15 & 0.57 & 0.15 & 0.43 & 0.25 & 1 & 0.43 & 0.25 & 0 & 0 & $i$ & 0 & 0 & 6 & 0.46 & 0.18 & 0.43 & 0.1 & - \\
\hline IBGX:T & $\operatorname{lbg} x_{-} T_{-} H L$ & 832 & 52 & 0 & 0 & 0.02 & 0.01 & 0.33 & 0.11 & 0.79 & 0.77 & 1 & 0.79 & 0.77 & NA & & NA & & & 17 & 0.08 & 0.1 & 0.37 & 0.16 & - \\
\hline IEGJ:P & le6j_P_HL & 210 & 12 & 0 & 0 & 0.08 & 0.03 & 1 & 0.41 & 0 & 0 & 9 & 0.42 & 0.24 & 0 & 0 & 7 & 0.42 & 0.26 & 5 & 0.33 & 0.08 & 0 & 0 & - \\
\hline$|E G|: A$ & leg_A_HL & 101 & 11 & 0.27 & 0.27 & 0.64 & 0.44 & 0.64 & 0.44 & 0.27 & 0.11 & 1 & 0.27 & 0.11 & 0.73 & 0.8 & 1 & 0.73 & 0.8 & 1 & 1 & 0.13 & 0.91 & 0.16 & * \\
\hline IFSK:A & Ifsk_A_CB & 159 & 17 & 0 & 0 & 0.59 & 0.17 & 0.59 & 0.17 & 0.59 & 0.31 & 1 & 0.59 & 0.31 & 0 & 0 & 8 & 0.47 & 0.47 & 6 & 0.12 & 0.11 & 0.76 & 0.22 & * \\
\hline IHOD:C & IhOd_C_BA & 123 & 17 & 0.65 & 0.85 & 0.06 & 0.05 & 0.59 & 1 & 0 & 0 & 10 & 0.53 & 0.5 & 0.59 & 0.56 & 1 & 0.59 & 0.56 & 5 & 0.44 & 0.16 & 0.35 & 0.13 & * \\
\hline |I9R:A & li9r_A_HL & 146 & 18 & 0 & 0 & 0 & 0 & 0 & 0 & 0.17 & 0.14 & 3 & 0.78 & 0.61 & 0.11 & 0.14 & 5 & 0.44 & 0.33 & 7 & 0.11 & 0.1 & 0.17 & 0.23 & - \\
\hline IIQD:C & liqd_C_BA & 156 & 16 & 0.19 & 0.23 & 0 & 0 & 0 & 0 & 0.31 & 0.14 & 5 & 0.94 & 0.83 & 0.38 & 0.32 & 1 & 0.38 & 0.32 & 5 & 0.07 & 0.04 & 0.56 & 0.3 & $*$ \\
\hline IJRH:I & ljrh_IHL & 108 & 15 & 0.07 & 0.1 & 0.67 & 0.56 & 0.67 & 0.56 & 0.53 & 0.31 & I & 0.53 & 0.31 & 0.47 & 0.78 & i & 0.47 & 0.78 & I & 0.73 & 0.15 & 0.6 & 0.26 & - \\
\hline ILK3:A & IIk3_A_HL & 160 & 18 & 0 & 0 & 0 & 0 & 0 & 0 & 0.11 & 0.1 & 2 & 0.67 & 0.57 & 0.22 & 0.27 & 5 & 0.56 & 0.62 & 5 & 0.17 & 0.14 & 0.61 & 0.32 & * \\
\hline IMHP:B & ImhP_B_XY & 192 & 19 & 0 & 0 & 0 & 0 & 0.47 & 0.33 & 0.74 & 0.61 & 1 & 0.74 & 0.61 & 0.68 & 0.76 & 1 & 0.68 & 0.76 & 2 & 0.11 & 0.13 & 0.53 & 0.27 & - \\
\hline INLO:G & InI0_G_HL & 51 & 7 & 0 & 0 & 0 & 0 & 0 & 0 & 0.29 & 0.25 & i & 0.29 & 0.25 & 0.2 & 0.07 & 2 & 1 & 0.5 & 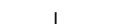 & 0.71 & 0.42 & 0.57 & 0.33 & - \\
\hline INSN:S & Insn_S_HL & 149 & 18 & 0 & 0 & 0 & 0 & 0.39 & 0.33 & 0.5 & 0.45 & i & 0.5 & 0.45 & 0 & 0 & 4 & 0.28 & 0.28 & 3 & 0.06 & 0.03 & 0.39 & 0.14 & - \\
\hline IOAZ:A & loaz_A_HL & 123 & 17 & 0.35 & 0.5 & 0.59 & 0.32 & 0.59 & 0.32 & 0.65 & 0.46 & 1 & 0.65 & 0.46 & 0.82 & 0.82 & i & 0.82 & 0.82 & 5 & 0.59 & 0.23 & 0.29 & 0.2 & $*$ \\
\hline IORQ:C & lorq_C_BA & 223 & 14 & 0 & 0 & 0 & 0 & 0.5 & 0.14 & 0 & 0 & 7 & 0.5 & 0.26 & 0.29 & 0.33 & I & 0.29 & 0.33 & 6 & 0.54 & 0.09 & 0 & 0 & - \\
\hline IORS:C & lors_C_BA & 132 & 10 & 0.6 & 0.46 & 0 & 0 & 0.7 & 0.3 & 0.2 & 0.08 & 4 & 0.6 & 0.24 & 0.4 & 0.24 & i & 0.4 & 0.24 & 4 & 0.78 & 0.11 & 0 & 0 & $*$ \\
\hline IPKQ:E & Ipkq_E_BA & 139 & 17 & 0.35 & 0.5 & 0.3 & 0.31 & 0.3 & 0.31 & 0.35 & 0.21 & 3 & 0.65 & 0.55 & 0.06 & 0.06 & 8 & 0.29 & 0.29 & 8 & 0.44 & 0.15 & 0.47 & 0.24 & - \\
\hline$|R| L L: C$ & Iril_C_BA & 95 & 13 & 0 & 0 & 0 & 0 & 0 & 0 & 0.31 & 0.19 & 6 & 0.69 & 0.39 & 0 & 0 & I & 0 & 0 & 5 & 0.58 & 0.14 & 0.54 & 0.23 & - \\
\hline ISYG:A & Isyb_A_HL & 204 & 11 & 0 & 0 & 0 & 0 & 0 & 0 & 0 & 0 & 1 & 0 & 0 & 0 & 0 & 3 & 0.45 & 0.24 & 8 & 0.3 & 0.1 & 0.91 & 0.14 & - \\
\hline ITZI:V & Itzi_V_BA & 102 & 4 & 0 & 0 & 0 & 0 & 0.75 & 0.3 & 0 & 0 & 1 & 0 & 0 & 0.5 & 0.09 & 6 & 0.75 & 0.14 & 3 & 1 & 0.05 & 0.5 & 0.05 & - \\
\hline IWEJ:F & Iwej_F_HL & 105 & 11 & 0 & 0 & 0 & 0 & 0 & 0 & 0.18 & 0.11 & 4 & 0.73 & 0.44 & 0.45 & 0.36 & 1 & 0.45 & 0.36 & 5 & 0.1 & 0.03 & 0.45 & 0.09 & - \\
\hline IYJD:C & Iyjd_C_HL & 140 & 14 & 0.14 & 0.17 & 0.5 & 0.64 & 0.5 & 0.64 & 0.57 & 0.36 & I & 0.57 & 0.36 & 0 & 0 & 4 & 0.64 & 0.32 & 6 & 0.36 & 0.11 & 0.21 & 0.16 & - \\
\hline IYNT:F & Iynt_F_BA & 254 & 19 & 0 & 0 & 0 & 0 & 0.79 & 0.58 & 0 & 0 & i & 0 & 0 & 0.74 & 0.88 & $i$ & 0.74 & 0.88 & 16 & 0.11 & 0.1 & 0 & 0 & - \\
\hline IYY9:A & Iyy9_A_DC & 624 & 20 & 0 & 0 & 0 & 0 & 0 & 0 & 0 & 0 & i & 0 & 0 & NA & & NA & & & 22 & 0 & 0 & 0.2 & 0.07 & - \\
\hline IZA3:R & |zza3_R_HL & 134 & 15 & 0.13 & 0.2 & 0.47 & 0.41 & 0.47 & 0.41 & 0.73 & 0.39 & 1 & 0.73 & 0.39 & 0 & 0 & 2 & 1 & 0.88 & 5 & 0.57 & 0.2 & 0.13 & 0.25 & - \\
\hline IZTX:E & IZtx_E_HL & 108 & 16 & 0.06 & 0.09 & 0 & 0 & 0 & 0 & 0.38 & 0.24 & & 0.44 & 0.37 & 0 & 0 & 3 & 0.56 & 0.45 & 1 & 0.75 & 0.16 & 0.19 & 0.21 & - \\
\hline 2JELL:P & 2jel_P_HL & 85 & 15 & 0 & 0 & 0 & 0 & 0 & 0 & 0 & 0 & 3 & 0.4 & 0.38 & 0 & 0 & 5 & 0.47 & 0.37 & 5 & 0.43 & 0.14 & 0.07 & 0.2 & - \\
\hline$|A| 4: N$ & lal4_N_HL & 388 & 17 & 0 & 0 & 0 & 0 & 0.35 & 0.18 & 0.18 & 0.12 & 6 & 0.47 & 0.33 & 0 & 0 & 1 & 0 & 0 & 11 & 0 & 0 & 0.76 & 0.2 & * \\
\hline$|A| 4: N$ & Inca_N_HL & $\begin{array}{l}380 \\
388\end{array}$ & 21 & 0 & 0 & 0 & 0 & 0.52 & 0.33 & $\begin{array}{l}0.10 \\
0\end{array}$ & $\begin{array}{c}.12 \\
0\end{array}$ & 4 & 1 & 0.81 & 0 & 0 & 5 & 0.86 & 0.86 & 11 & 0 & 0 & 0.62 & 0.2 & $*$ \\
\hline $\mid \mathrm{RJ} C \mathrm{C}: \mathrm{B}$ & Ibvk_C_BA & 129 & 17 & 0 & 0 & 0.12 & 0.09 & 0.47 & 0.47 & 0.06 & 0.05 & i & 0.06 & 0.05 & 0 & 0 & 3 & 0.76 & 0.65 & 3 & 0.24 & 0.1 & 0.29 & 0.23 & * \\
\hline IRJC:B & Ijhl_A_HL & 129 & 11 & 0 & 0 & 0.27 & 0.13 & 0.27 & 0.13 & 0 & 0 & 2 & 0.82 & 0.36 & 0 & 0 & 5 & 0.45 & 0.33 & 3 & 0.1 & 0.03 & 0.27 & 0.14 & * \\
\hline IRJC:B & Indg_C_BA & 129 & 21 & 0.29 & 0.46 & 0.38 & 0.35 & 0.38 & 0.35 & 0.57 & 0.55 & 1 & 0.57 & 0.55 & 0 & 0 & 2 & 0.33 & 0.33 & 3 & 0.43 & 0.23 & 0.33 & 0.32 & $*$ \\
\hline IRJC:B & IP2C_C_BA & 129 & 18 & 0.11 & 0.15 & 0.17 & 0.13 & 0.17 & 0.13 & 0.28 & 0.25 & 9 & 0.33 & 0.33 & 0.17 & 0.21 & 2 & 0.67 & 0.6 & 3 & 0.56 & 0.26 & 0.5 & 0.41 & * \\
\hline IJPS:T & Ijps___HL & 219 & 21 & 0.05 & 0.1 & 0.14 & 0.09 & 0.14 & 0.09 & 0.05 & 0.04 & 2 & 0.57 & 0.32 & 0.86 & 0.9 & 1 & 0.86 & 0.9 & 7 & 0.25 & 0.13 & 0.33 & 0.19 & $*$ \\
\hline IARI:B & larl_B_CD & 298 & 16 & 0 & 0 & 0.06 & 0.03 & 0.06 & 0.03 & 0.06 & 0.04 & 1 & 0.06 & 0.04 & 0 & 0 & 1 & 0 & 0 & 12 & 0.13 & 0.05 & 0 & 0 & * \\
\hline IEOB:A & leo8_A_HL & 328 & 15 & 0 & 0 & 0 & 0 & 0.87 & 0.23 & 0 & 0 & 1 & 0 & 0 & 0 & 0 & i & 0 & 0 & 14 & 0.07 & 0.03 & 0 & 0 & $*$ \\
\hline |EOB:A & Iken_A_HL & 328 & 16 & 0 & 0 & 0.69 & 0.23 & 0.69 & 0.23 & 0 & 0 & I & 0 & 0 & 0 & 0 & I & 0 & 0 & 14 & 0.13 & 0.06 & 0.56 & 0.13 & - \\
\hline |EOB:A & Iffu A HL & 328 & 19 & 0 & 0 & 0 & 0 & 0.84 & 0.29 & 0 & 0 & 7 & 0.21 & 0.17 & 0 & 0 & 3 & 0.21 & 0.27 & 14 & 0.11 & 0.05 & 0.11 & 0.03 & $*$ \\
\hline IEOB:A & 2vit_C_BA & 328 & 18 & 0 & 0 & 0.33 & 0.13 & 0.33 & 0.13 & 0.22 & 0.02 & 2 & 1 & 0.1 & 0 & 0 & 10 & 0.22 & 0.33 & 14 & 0.18 & 0.08 & 0.17 & 0.04 & . \\
\hline IEZV:E & lezv_E_XY & 185 & 17 & 0 & 0 & 0 & 0 & 0 & 0 & 0.18 & 0.09 & 7 & 0.53 & 0.53 & 0 & 0 & 1 & 0 & 0 & 7 & 0.31 & 0.07 & 1 & 0.29 & $*$ \\
\hline IOSP:O & Iosp_O_HL & 257 & 19 & 0 & 0 & 0.05 & 0.02 & 0.05 & 0.02 & 0 & 0 & 4 & 0.63 & 0.31 & 0 & 0 & i & 0 & 0 & 14 & 0.17 & 0.07 & 0.53 & 0.17 & $*$ \\
\hline IOSP:O & IfILF_BA & 257 & 17 & 0 & 0 & 0 & 0 & 0.71 & 0.24 & 0 & 0 & 5 & 0.59 & 0.3 & 0.29 & 0.56 & i & 0.29 & 0.56 & 14 & 0.25 & 0.09 & 0.47 & 0.14 & $*$ \\
\hline IFNS:A & Iffs__A_HL & 196 & 12 & 0 & 0 & 0 & 0 & 0 & 0 & 0.17 & 0.07 & 6 & 0.42 & 0.21 & 0 & 0 & 8 & 0.33 & 0.22 & 7 & 0 & 0 & 0.67 & 0.3 & * \\
\hline IG9M:G & Ig9m_G_HL & 321 & 12 & 0 & 0 & 0.67 & 0.12 & 0.67 & 0.12 & 0.08 & 0.02 & 2 & 0.33 & 0.1 & 0.5 & 0.29 & 1 & 0.5 & 0.29 & 14 & 0.18 & 0.05 & 0.08 & 0.01 & $*$ \\
\hline IG9M:G & 2b4c_G_HL & 321 & 17 & 0 & 0 & 0.75 & 0.13 & 0.75 & 0.13 & 0.71 & 0.21 & 1 & 0.71 & 0.21 & 0.29 & 0.21 & 1 & 0.29 & 0.21 & 14 & 0.09 & 0.03 & 0.08 & 0.01 & - \\
\hline $\mid R 3\}: C$ & Ir3i_C_BA & 124 & 13 & 0 & 0 & 0 & 0 & 0 & 0 & 0.31 & 0.15 & 5 & 0.85 & 0.85 & 0.85 & 0.92 & 1 & 0.85 & 0.92 & 4 & 0.42 & 0.11 & 0.08 & 0.09 & * \\
\hline IN8Z:C & In8z_C_BA & 607 & 17 & 0.24 & 0.07 & 0.3 & 0.38 & 0.3 & 0.38 & 0.24 & 0.09 & 1 & 0.24 & 0.09 & NA & & NA & & & 18 & 0.18 & 0.1 & 0.12 & 0.05 & $*$ \\
\hline IN8Z:C & Is78_B_FE & 607 & 23 & 0 & 0 & 0 & 0 & 0 & 0 & 0 & 0 & i & 0 & 0 & NA & & NA & & & 18 & 0.05 & 0.03 & 0.22 & 0.12 & - \\
\hline INFD:D & Inff_D_HG & 239 & 13 & 0 & 0 & 0 & 0 & 0.92 & 0.32 & 0.15 & 0.06 & i & 0.15 & 0.06 & 0 & 0 & 5 & 0.31 & 0.18 & 13 & 0.25 & 0.08 & 0.77 & 0.16 & $*$ \\
\hline ITQB:A & Itqb_A_BC & 102 & 18 & 0 & 0 & 0.28 & 0.29 & 0.67 & 0.57 & 0.56 & 0.53 & 1 & 0.56 & 0.53 & 0.17 & 0.2 & 3 & 0.5 & 0.53 & 2 & 0.11 & 0.08 & 0.78 & 0.21 & $*$ \\
\hline ITXV:A & Itov_A_HL & 452 & 19 & 0 & 0 & 0 & 0 & 0 & 0 & 0 & 0 & i & 0 & 0 & 0 & 0 & 7 & 0.53 & 0.53 & 18 & 0.11 & 0.06 & 0.53 & 0.17 & $*$ \\
\hline IV7M:V & IV7m_V_HL & 163 & 17 & 0 & 0 & 0 & 0 & 0.35 & 0.32 & 0.41 & 0.39 & 1 & 0.41 & 0.39 & 0.35 & 0.38 & i & 0.35 & 0.38 & 6 & 0.31 & 0.15 & 0.06 & 0.11 & . \\
\hline IXIW:A & |xin_A_DC & 105 & 18 & 0 & 0 & 0 & 0 & 0 & 0 & 1 & 0.86 & $\mathrm{i}$ & 1 & 0.86 & 0.83 & 0.79 & i & 0.83 & 0.79 & 26 & 0 & 0 & 0.88 & 0.43 & - \\
\hline IXIW:F & Ixiw_E_DC & 79 & 10 & 0.1 & 0.14 & 0 & 0 & 0 & 0 & 0.1 & 0.05 & 8 & 0.6 & 0.32 & 0.1 & 0.07 & i & 0.1 & 0.07 & 26 & 0 & 0 & 0.4 & 0.44 & - \\
\hline IZ3G:A & Iz3g_A_HL & 186 & 19 & 0.12 & 0.11 & 0.35 & 0.17 & 0.35 & 0.17 & 0.53 & 0.3 & 1 & 0.53 & 0.3 & 0 & 0 & 8 & 0.26 & 0.25 & 10 & 0.25 & 0.11 & 0.35 & 0.35 & - \\
\hline 2AEP:A & 2aep_A_HL & 395 & 21 & 0 & 0 & 0 & 0 & 0.48 & 0.3 & 0 & 0 & 1 & 0 & 0 & 0.05 & 0.05 & 1 & 0.05 & 0.05 & 18 & 0.1 & 0.07 & 0.14 & 0.05 & - \\
\hline IROA:B & IrO__B_HL & 429 & 11 & & & 0.73 & 0.08 & 0.73 & 0.08 & 0.36 & 0.15 & 1 & 0.36 & 0.15 & 0 & 0 & 1 & 0 & 0 & 10 & 0 & 0 & 1 & 0.06 & $*$ \\
\hline
\end{tabular}


Positive predictive value $(\mathrm{PPV})($ precision $)=T P /(T P+F P)$ - a proportion of correctly predicted epitope residues (TP) with respect to the total number of predicted epitope residues $(\mathrm{TP}+\mathrm{FN})$.

Accuracy $($ ACC $)=(T P+T N) /(T P+F N+F P+T N)-$ a proportion of correctly predicted epitope and non-epitope residues with respect to all residues.

Area under the ROC Curve (AUC) - A ROC curve is a graph representing a dependency of TPR versus FPR, or sensitivity versus specificity. The AUC measure is a widely used measure for immunoinformatics and bioinformatics methods; it has also been recommended for methods comparison in the recent report [25]. The AUC gives the general performance of the method; it is "equivalent to the probability that the classifier will rank a randomly chosen positive instance higher than a randomly chosen negative instance" [61].

Success Rate - the number of epitopes from the dataset that were successfully predicted. While the AUC is a convenient and commonly used measure in immunoinformatics since many protein-protein binding site prediction methods, as well as three methods evaluated in the current work, ProMate, PPI-PRED, and CEP, used success rate as a measure of their performance, we considered it necessary to also calculate the methods success rates. While this measure is easily and naturally interpretable, it requires us to define the successful prediction and that can be done in many different ways. For this reason, many scientists try to avoid using this measure.

The statistical significance of a prediction, that is, the difference between observed and expected frequencies of an actual epitope/non-epitope residue in the predicted epitope/non-epitope, was determined by Fisher's exact test (right-tailed). The prediction was considered significant if the significance level was $\geq 95 \%$, that is, the P-value was $\leq 0.05$.

The above parameters were applied in evaluating the methods as follows:

(1) For the scale-based methods, ProMate, DiscoTope, ConSurf, and PIER, by varying the threshold values for score values classifying epitope residues from non-epitope residues, the AUC values have been calculated for each epitope.

(2) Success rates for all methods were calculated on a per protein bases taking into account one epitope per protein predicted with the highest significance. Such an approach assumes that if the epitope in a protein was successfully predicted, the prediction for the protein is successful. Cri- teria used for definition of successful prediction are discussed further.

(3) Patch prediction methods and protein-protein docking methods fall in the category of discrete classifiers, that is, they classify a residue as an epitope or non-epitope residue with no score assigned. Therefore, a ROC curve cannot be generated for these methods, only the AUC value can be estimated. Other statistics have also been obtained for these methods by averaging statistical values over epitopes and then calculating the overall statistical values over epitope and non-epitope residues in the dataset.

\section{Prediction of individual epitopes}

The results for each method in predicting 59 representative epitopes are given in Tables 2, 3 and supplementary materials [see Additional file 2]. For scale-based methods only the AUC values were computed (Table 3 ), while for patch prediction and docking methods all other statistics were produced (Table 2 and supplementary materials [see Additional file 2]).

DiscoTope and ProMate predict only one epitope per protein. ClusPro and PatchDock rank predicted models starting from the model with the best score. For these methods, the first (by rank) prediction was considered. If it was not significant $(p>0.05)$, the next by rank significant prediction (not exceeding the 10 best predictions) was reported in Table 2. Since the number of epitopes predicted by CEP in a protein varies (Table 2) and they are not ranked, the average prediction was reported for each epitope. More detailed statistics on the prediction results is provided in the supplementary table [see Additional file 2].

No one epitope was predicted by all methods (Table 2). Some epitopes, for example, HyHEL-8 on HEL [PDB:1NDG] and 8-18C5 on myelin oligodendrocyte glycoprotein [PDB:1PKQ], were predicted by all methods except CEP (Table 2). Two epitopes, cetuximab on EGFR [PDB:1YY9] and 7E2C50S on cytochrome c oxidase [PDB:1AR1], appeared to be difficult to predict; they could probably be predicted using the ConSurf average score in combination with a patch generation method. The extracellular region of EGFR [PDB:1YY9] is a large (624 aa) loosely-packed multi-domain protein with a lot of loops and hence epitope recognition appears difficult. Similarly, recognition of epitopes on subunit II of cytochrome $\mathrm{c}$ oxidase [PDB:1AR1] appears problematic because the protein possesses long protruded $\alpha$-helixes.

The lower specificity of CEP and DiscoTope [see Additional file 2] results from these methods predicting larger epitopes (average size of predicted epitope by CEP is 40 residues, DiscoTope (-7.7) - 43 and DiscoTope (-10.5) - 
Table 3: AUC values for representative epitopes

\begin{tabular}{|c|c|c|c|c|c|c|c|c|c|c|c|c|c|c|c|c|}
\hline antigen & epitope & PIER & ConSurf & $\begin{array}{c}\text { ConSurf } \\
\text { (confidence score) }\end{array}$ & $\begin{array}{l}\text { Promate } \\
\text { (score) }\end{array}$ & $\begin{array}{l}\text { ProMate } \\
\text { (patch) }\end{array}$ & $\begin{array}{l}\text { PPI-PRED } \\
\text { (Ist patch) }\end{array}$ & $\begin{array}{l}\text { PPI-PRED } \\
\text { (best patch) }\end{array}$ & $\begin{array}{l}\text { PatchDock } \\
\text { Ist model }\end{array}$ & $\begin{array}{l}\text { PatchDock } \\
\text { best model of } 10\end{array}$ & $\begin{array}{c}\text { ClusPro (DOT) } \\
\text { Ist model }\end{array}$ & $\begin{array}{l}\text { ClusPro (DOT) } \\
\text { best model of I0 }\end{array}$ & CEP & $\begin{array}{l}\text { DiscoTope } \\
\text { (score) }\end{array}$ & $\begin{array}{c}\text { DiscoTope } \\
(-7.7)\end{array}$ & $\begin{array}{l}\text { Is in training } \\
\text { set?\& }\end{array}$ \\
\hline 2ADF:A & 2adf_A_HL & 0.01 & 0.62 & 0.62 & 0.88 & NA & 0.88 & 0.88 & 0.45 & 0.66 & 0.81 & 0.81 & 0.57 & 0.53 & 0.51 & - \\
\hline 2ADF:A & Ife8_B_IM & 0.16 & 0.67 & 0.67 & 0.65 & NA & 0.62 & 0.62 & 0.45 & 0.67 & 0.79 & 0.79 & 0.60 & 0.81 & 0.56 & $*$ \\
\hline IAFV:A & lafv_A_HL & 0.78 & 0.48 & 0.41 & 0.60 & 0.49 & 0.62 & 0.62 & 0.65 & 0.65 & 0.43 & 0.43 & 0.63 & 0.53 & 0.52 & - \\
\hline IBGX:T & Ibgx_T_HL & 0.39 & 0.48 & 0.50 & 0.56 & NA & 0.42 & 0.58 & 0.89 & 0.89 & NA & NA & 0.52 & 0.74 & 0.62 & - \\
\hline IE6):P & le6j_P_HL & 0.51 & 0.43 & 0.39 & 0.41 & NA & 0.46 & 0.96 & 0.43 & 0.67 & 0.47 & 0.67 & 0.55 & 0.23 & 0.30 & - \\
\hline IEGJ:A & legi_A_HL & 0.09 & NA & NA & 0.84 & 0.59 & 0.77 & 0.77 & 0.50 & 0.50 & 0.85 & 0.85 & 0.61 & 0.88 & 0.67 & * \\
\hline IFSK:A & Ifsk_A_CB & 0.85 & 0.31 & 0.33 & 0.54 & 0.44 & 0.62 & 0.62 & 0.72 & 0.72 & 0.44 & 0.70 & 0.50 & 0.82 & 0.71 & $*$ \\
\hline IHOD:C & IhOd_C_BA & 0.25 & 0.51 & 0.51 & 0.97 & 0.82 & 0.43 & 0.80 & 0.38 & 0.73 & 0.76 & 0.76 & 0.55 & 0.50 & 0.49 & * \\
\hline II9R:A & ligr_A_HL & 0.47 & 0.72 & 0.74 & 0.43 & 0.45 & 0.45 & 0.45 & 0.51 & 0.86 & 0.51 & 0.66 & 0.48 & 0.71 & 0.55 & - \\
\hline IIQD:C & liqd_C_BA & 0.10 & 0.80 & 0.81 & 0.74 & 0.56 & 0.44 & 0.44 & 0.55 & 0.96 & 0.64 & 0.64 & 0.45 & 0.78 & 0.71 & $*$ \\
\hline IJRH:I & Ijph_I_HL & 0.57 & 0.62 & NA & 0.49 & 0.49 & 0.79 & 0.79 & 0.67 & 0.67 & 0.72 & 0.72 & 0.53 & 0.62 & 0.67 & - \\
\hline ILK3:A & IIk3_A_HL & 0.71 & 0.72 & 0.76 & 0.38 & 0.45 & 0.40 & 0.40 & 0.49 & 0.81 & 0.57 & 0.76 & 0.52 & 0.81 & 0.72 & * \\
\hline IMHP:B & ImhP_B_XY & 0.15 & 0.44 & 0.42 & 0.88 & NA & 0.42 & 0.68 & 0.85 & 0.85 & 0.83 & 0.83 & 0.52 & 0.81 & 0.69 & - \\
\hline INLO:G & InlO_G_HL & 0.23 & NA & NA & 0.16 & 0.45 & 0.42 & 0.42 & 0.58 & 0.58 & 0.46 & 0.95 & 0.78 & 0.61 & 0.69 & - \\
\hline INSN:S & Insn_S_HL & 0.76 & 0.75 & 0.78 & 0.26 & 0.45 & 0.40 & 0.64 & 0.71 & 0.71 & 0.44 & 0.59 & 0.41 & 0.58 & 0.53 & - \\
\hline IOAZ:A & loaz_A_HL & 0.17 & 0.33 & 0.25 & 0.85 & 0.65 & 0.70 & 0.70 & 0.77 & 0.77 & 0.90 & 0.90 & 0.64 & 0.61 & 0.55 & * \\
\hline IORQ:C & lorq_C_BA & 0.65 & 0.60 & 0.61 & 0.55 & 0.44 & 0.33 & 0.64 & 0.42 & 0.70 & 0.63 & 0.63 & 0.60 & 0.48 & 0.44 & - \\
\hline IORS:C & lors_C_BA & 0.59 & NA & NA & 0.96 & 0.77 & 0.39 & 0.78 & 0.51 & 0.72 & 0.65 & 0.65 & 0.66 & 0.50 & 0.42 & $*$ \\
\hline IPKQ:E & Ipkq_E_BA & 0.48 & 0.69 & 0.70 & 0.76 & 0.65 & 0.60 & 0.60 & 0.59 & 0.79 & 0.46 & 0.60 & 0.56 & 0.71 & 0.63 & - \\
\hline IRJLLCC & Irjl_C_BA & 0.64 & 0.51 & 0.48 & 0.33 & 0.48 & 0.37 & 0.37 & 0.55 & 0.76 & 0.39 & 0.39 & 0.53 & 0.73 & 0.62 & - \\
\hline ISYG:A & Isy6_A_HL & 0.83 & 0.45 & NA & 0.54 & 0.49 & 0.43 & 0.43 & 0.43 & 0.43 & 0.45 & 0.68 & 0.58 & 0.82 & 0.80 & - \\
\hline ITZI:V & Itzi_V_BA & 0.20 & 0.49 & 0.55 & 0.56 & 0.45 & 0.37 & 0.84 & 0.36 & 0.36 & 0.64 & 0.78 & 0.60 & 0.52 & 0.54 & - \\
\hline IWEJ:F & Iwej_F_HL & 0.67 & 0.82 & 0.83 & 0.33 & 0.44 & 0.45 & 0.45 & 0.50 & 0.81 & 0.68 & 0.68 & 0.39 & 0.47 & 0.45 & - \\
\hline IYJD:C & Iyjd_C_HL & 0.44 & 0.53 & 0.54 & 0.80 & 0.53 & 0.73 & 0.73 & 0.73 & 0.73 & 0.44 & 0.74 & 0.52 & 0.58 & 0.54 & . \\
\hline IYNT:F & Iynt_F_BA & 0.31 & NA & NA & 0.69 & NA & 0.44 & 0.87 & 0.44 & 0.44 & 0.87 & 0.87 & 0.51 & 0.49 & 0.46 & - \\
\hline IYY9:A & Iyy9_A_DC & 0.46 & 0.74 & 0.75 & 0.20 & 0.45 & 0.46 & 0.46 & 0.47 & 0.47 & NA & NA & 0.48 & 0.68 & 0.55 & - \\
\hline IZA3:R & Iza3_R_HL & 0.29 & 0.72 & 0.77 & 0.61 & 0.53 & 0.69 & 0.69 & 0.80 & 0.80 & 0.45 & 0.99 & 0.65 & 0.69 & 0.54 & - \\
\hline IZTX:E & Iztx_E_HL & 0.73 & 0.63 & 0.63 & 0.37 & 0.48 & 0.43 & 0.43 & 0.59 & 0.66 & 0.39 & 0.72 & 0.54 & 0.47 & 0.54 & - \\
\hline 2JELLP & 2jel_P_HL & 0.58 & 0.70 & 0.70 & 0.59 & NA & 0.41 & 0.41 & 0.36 & 0.63 & 0.40 & 0.65 & 0.44 & 0.66 & 0.51 & - \\
\hline$|A| 4: N$ & lal4_N_HL & 0.69 & 0.75 & 0.76 & 0.38 & 0.45 & 0.46 & 0.64 & 0.56 & 0.72 & 0.47 & 0.47 & 0.47 & 0.87 & 0.81 & * \\
\hline |A|4:N & Inca_N_HL & 0.56 & 0.67 & 0.69 & 0.30 & 0.45 & 0.46 & 0.73 & 0.46 & 1.00 & 0.47 & 0.93 & 0.47 & 0.84 & 0.74 & $*$ \\
\hline IRJC:B & Ibvk_C_BA & 0.61 & 0.62 & 0.61 & 0.66 & 0.44 & 0.47 & 0.69 & 0.44 & 0.44 & 0.44 & 0.85 & 0.46 & 0.66 & 0.57 & * \\
\hline IRJC:B & Ijhl_A_HL & 0.45 & 0.73 & 0.73 & 0.51 & 0.44 & 0.55 & 0.55 & 0.42 & 0.84 & 0.44 & 0.68 & 0.39 & 0.79 & 0.55 & $*$ \\
\hline IRJC:B & Indg_C_BA & 0.46 & 0.66 & 0.65 & 0.66 & 0.61 & 0.62 & 0.62 & 0.74 & 0.74 & 0.40 & 0.60 & 0.57 & 0.74 & 0.60 & $*$ \\
\hline IRJC:B & IP2c_C_BA & 0.70 & 0.55 & 0.57 & 0.48 & 0.51 & 0.49 & 0.49 & 0.57 & 0.61 & 0.54 & 0.80 & 0.65 & 0.74 & 0.69 & * \\
\hline IJPS:T & Ijps_T_HL & 0.49 & 0.63 & 0.72 & 0.77 & 0.50 & 0.49 & 0.49 & 0.46 & 0.72 & 0.92 & 0.92 & 0.54 & 0.62 & 0.59 & * \\
\hline IARI:B & larl_B_CD & 0.87 & 0.62 & 0.64 & 0.15 & 0.46 & 0.48 & 0.48 & 0.49 & 0.49 & 0.47 & 0.47 & 0.49 & 0.57 & 0.45 & * \\
\hline IEO8:A & leo8_A_HL & 0.43 & 0.64 & 0.65 & 0.67 & 0.49 & 0.42 & 0.87 & 0.45 & 0.45 & 0.48 & 0.48 & 0.48 & 0.27 & 0.39 & $*$ \\
\hline IEOQ:A & Iken_A_HL & 0.32 & 0.61 & 0.62 & 0.59 & 0.49 & 0.79 & 0.79 & 0.44 & 0.44 & 0.49 & 0.49 & 0.51 & 0.76 & 0.68 & - \\
\hline IEOQ:A & Iqfu_A_HL & 0.54 & 0.64 & 0.64 & 0.60 & 0.49 & 0.42 & 0.86 & 0.45 & 0.58 & 0.48 & 0.59 & 0.50 & 0.38 & 0.44 & * \\
\hline IEOQ:A & 2vit_C_BA & 0.51 & 0.56 & 0.63 & 0.48 & 0.49 & 0.60 & 0.60 & 0.29 & 0.68 & 0.48 & 0.59 & 0.54 & 0.58 & 0.48 & - \\
\hline IEZV:E & lezv_E_XY & 0.84 & 0.62 & 0.64 & 0.23 & 0.44 & 0.42 & 0.42 & 0.50 & 0.74 & 0.48 & 0.48 & 0.47 & 0.85 & 0.88 & $*$ \\
\hline IOSP:O & losp_O_HL & 0.90 & 0.41 & 0.38 & 0.82 & NA & 0.40 & 0.40 & 0.46 & 0.76 & 0.48 & 0.48 & 0.49 & 0.76 & 0.66 & * \\
\hline IOSP:O & IfjI_F_BA & 0.17 & 0.50 & 0.50 & 0.62 & NA & 0.37 & 0.77 & 0.45 & 0.75 & 0.64 & 0.64 & 0.54 & 0.68 & 0.63 & * \\
\hline IFNS:A & Ifns_A_HL & 0.50 & 0.57 & 0.57 & 0.40 & NA & 0.45 & 0.45 & 0.52 & 0.66 & 0.45 & 0.63 & 0.44 & 0.92 & 0.78 & $*$ \\
\hline IG9M:G & $\lg 9 \mathrm{~m} \_\mathrm{G} \_\mathrm{HL}$ & 0.17 & 0.49 & 0.48 & 0.68 & 0.45 & 0.74 & 0.74 & 0.47 & 0.61 & 0.73 & 0.73 & 0.53 & 0.44 & 0.43 & * \\
\hline IG9M:G & 2b4c_G_HL & 0.13 & 0.46 & 0.44 & 0.68 & 0.45 & 0.78 & 0.78 & 0.79 & 0.79 & 0.62 & 0.62 & 0.48 & 0.43 & 0.43 & - \\
\hline$|R 3|: C$ & $\operatorname{lr} 3 \mathrm{j}_{\mathrm{C}} \mathrm{C} C \mathrm{BA}$ & 0.84 & 0.81 & 0.81 & 0.53 & 0.45 & 0.42 & 0.42 & 0.56 & 0.92 & 0.92 & 0.92 & 0.52 & 0.72 & 0.49 & * \\
\hline IN8Z:C & In8z_C_BA & 0.30 & 0.46 & 0.46 & 0.82 & 0.57 & 0.64 & 0.64 & 0.59 & 0.59 & NA & NA & 0.57 & 0.59 & 0.53 & $*$ \\
\hline IN8Z:C & Is78_B_FE & 0.16 & 0.56 & 0.57 & 0.60 & 0.45 & 0.49 & 0.49 & 0.47 & 0.47 & NA & NA & 0.50 & 0.55 & 0.58 & - \\
\hline INFD:D & Infd_D_HG & 0.90 & 0.73 & 0.71 & 0.34 & 0.46 & 0.43 & 0.90 & 0.51 & 0.51 & 0.46 & 0.62 & 0.55 & 0.88 & 0.77 & $*$ \\
\hline ITQB:A & Itqb_A_BC & 0.44 & 0.42 & 0.44 & 0.33 & 0.43 & 0.57 & 0.78 & 0.73 & 0.73 & 0.51 & 0.70 & 0.41 & 0.59 & 0.57 & * \\
\hline ITXV:A & Itxv_A_HL & 0.64 & 0.88 & 0.89 & 0.59 & 0.45 & 0.43 & 0.43 & 0.47 & 0.47 & 0.47 & 0.75 & 0.52 & 0.87 & 0.71 & $*$ \\
\hline IV7M:V & Iv7m_V_HL & 0.67 & 0.59 & NA & 0.37 & 0.48 & 0.45 & 0.63 & 0.67 & 0.67 & 0.64 & 0.64 & 0.56 & 0.47 & 0.50 & - \\
\hline IXIW:A & Ixiw_A_DC & 0.85 & 0.76 & 0.90 & 0.31 & 0.44 & 0.41 & 0.41 & 0.99 & 0.99 & 0.89 & 0.89 & 0.45 & 0.87 & 0.83 & . \\
\hline IXIW:F & Ixiw_F_DC & 0.69 & 0.65 & 0.74 & 0.60 & 0.51 & 0.41 & 0.41 & 0.42 & 0.71 & 0.45 & 0.45 & 0.48 & 0.59 & 0.66 & - \\
\hline IZ3G:A & |z3g_A_HL & 0.48 & 0.65 & 0.59 & 0.34 & 0.51 & 0.59 & 0.59 & 0.70 & 0.70 & 0.43 & 0.59 & 0.53 & 0.66 & 0.64 & . \\
\hline 2AEP:A & 2aep_A_HL & 0.68 & 0.50 & 0.51 & 0.43 & 0.45 & 0.46 & 0.71 & 0.46 & 0.46 & 0.50 & 0.50 & 0.52 & 0.70 & 0.49 & - \\
\hline IROA:B & IrOa_B_HL & 0.12 & 0.51 & 0.54 & 0.02 & NA & 0.76 & 0.76 & 0.65 & 0.65 & 0.47 & 0.47 & 0.45 & 0.94 & 0.79 & * \\
\hline
\end{tabular}

'NA' means that results for the epitope/protein were not obtained.

\& - Epitopes used in the DiscoTope training set are indicated by an asterisk; those not used in the training set are indicated by a hyphen. 
80 residues) in comparison with other methods. The average size of predicted epitope size for PatchDock is 29 residues, ClusPro (DOT) is 17 residues, and PPI-PRED is 32 residues. The size of actual epitopes in the dataset varies from 4 to 52 residues ( $\bar{x}=16, s=6$ ). However, it should be emphasized that if the most of the methods considered were designed to predict an epitope as a whole single entity, DiscoTope focuses on the prediction of individual epitope residues that can be part of several different epitopes in the same protein. Therefore, the average size of the epitope predicted by DiscoTope is large; moreover, the predicted epitope residues can be located too far from each on the protein surface to form a single epitope.

\section{Overall performance of each method}

The overall performance of each method have been compared first using average AUC values for all methods and then calculating all other statistics for patch prediction and protein-protein docking methods. Both comparisons were made on different subsets of representative epitopes from dataset \#2.
Calculating AUC values for all methods, we discarded from the analysis the proteins for which any method didn't produced a result (ConSurf, ProMate, and ClusPro (DOT) were not able to predict epitopes for several proteins, see Methods). The final subset contained 42 epitopes from Table 2 of which 21 epitopes were not used for DiscoTope training. All other methods didn't use any epitopes for training.

AUC values averaged on subsets of 42 and 21 epitopes are shown in Figure 8. ConSurf, DiscoTope, PPI-PRED and docking methods, when the 10 best models were considered, demonstrated average AUC values above 0.6, that is, poor or mediocre performance. PatchDock was the best, giving an AUC of 0.69. All other methods performed close to random (Fig. 8). DiscoTope gave AUC values of 0.65 and 0.62 on all representative epitopes and those that were not used by the method for training, respectively. When DiscoTope performance was evaluated by the authors of the method [49], it gave an AUC value of 0.71 averaged over the five evaluation sets used for cross-validation.

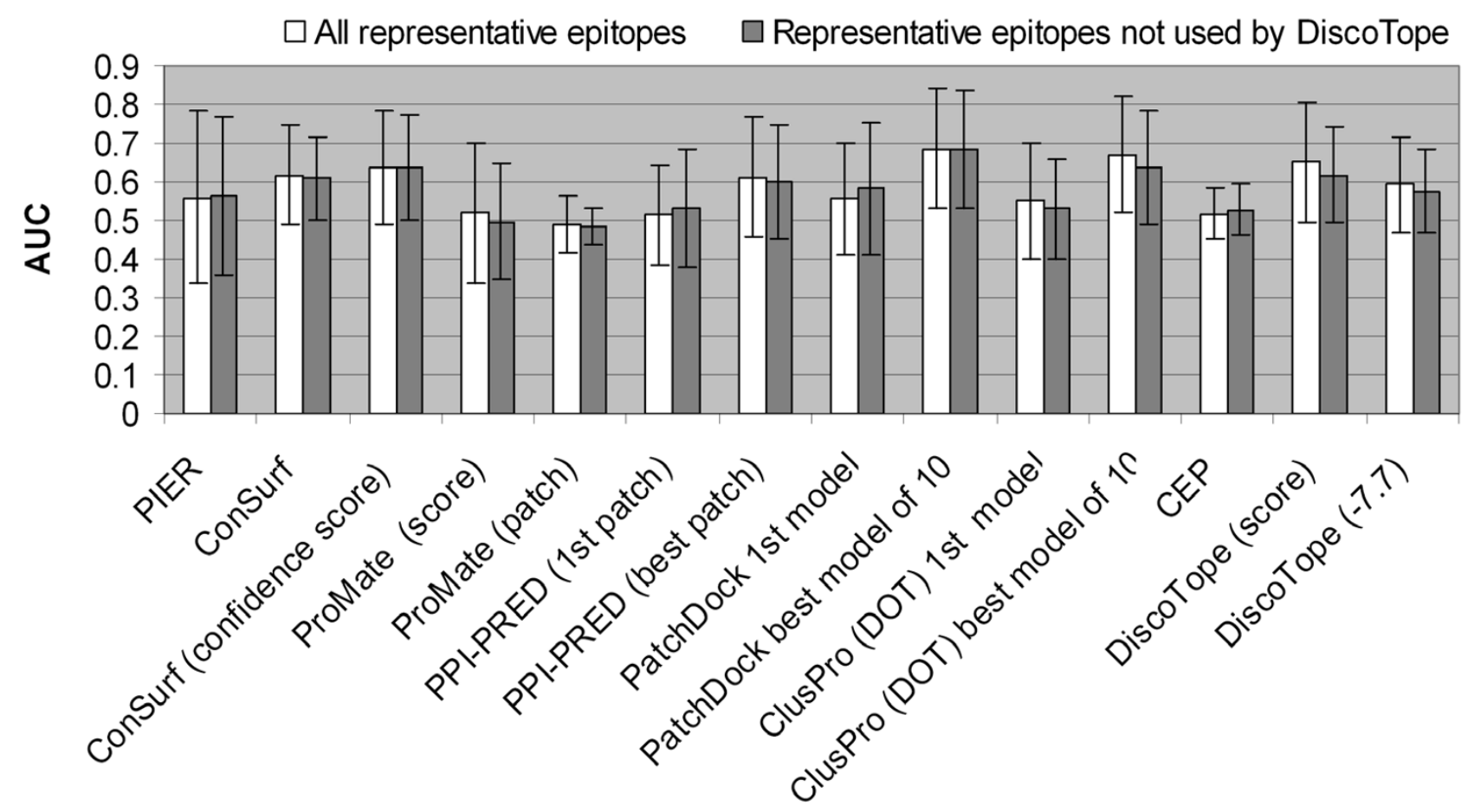

Figure 8

Average AUC values for each method. Vertical bars show one standard deviation. 
For patch prediction and docking methods, to avoid the problematic comparison of methods predicting one epitope per protein with those that predict several epitopes, all epitopes from proteins with more than one epitope have been removed from dataset \#2. Epitopes from proteins for which any method did not produce the prediction have also been discarded. The following statistics were calculated on the resulting subset of epitopes.

First, $\mathrm{FP}, \mathrm{FN}, \mathrm{TP}$, and $\mathrm{TN}$ values were summarized for the whole pool of epitopes, and sensitivity, specificity, accuracy, PPV, and AUC values calculated for each method (Table 4, upper part). AUC values obtained in this way were close to those demonstrated in Fig. 9. The best performers were docking methods PatchDock and DOT when the top ten models were considered, giving AUC values of 0.66 and 0.69 , respectively (Table 4 ). Among the methods producing one prediction per protein, DiscoTope was rated the best by with an AUC of 0.60.

Second, statistics were averaged over epitopes (Table 4, lower part). The overall performance was poor for all methods. The best performance demonstrated by docking methods (when the 10 best models were considered) was $41 \%$ PPV (precision) and 46\% sensitivity (recall) for ClusPro(DOT) and 30\% PPV and $42 \%$ sensitivity for PatchDock. Among the methods producing one prediction per protein, DiscoTope was rated the best by sensitivity (43\% sensitivity at $18 \%$ PPV) and ClusPro(DOT) first model by PPV (25\% sensitivity and 25\% PPV) (Fig. 9).

\section{Comparison of success rates}

Since patch prediction methods used in the current analysis used success rate as a performance measure, we additionally calculated the methods success rate on the subset of 42 epitopes used for overall methods comparison above. The prediction of each epitope was deemed suc- cessful if the AUC value was above a threshold value of 0.6 or 0.7. The results are presented in Fig. 10.

The proportion of epitopes predicted with an AUC $\geq 0.6$ for the scale-based methods (PIER, ConSurf, ProMate, and DiscoTope) and the methods providing only one prediction per protein (ProMate, DiscoTope, PPI-PRED first prediction, PatchDock first model and ClusPro(DOT) first model) was lower than 50\% except for ConSurf and DiscoTope methods, which showed success rates of approximately $60 \%$ (white bars in Fig. 10). Among the methods predicting several epitopes per protein (PPI-PRED, PatchDock, ClusPro(DOT), and CEP) PatchDock performed best with $>75 \%$ successful predictions at an AUC $\geq 0.6$ and $55 \%$ at an AUC $\geq 0.7$ (Fig. 10).

PPI-PRED predicted $75 \%$ of protein-protein binding interfaces successfully, with a specificity over $50 \%$ and sensitivity over $20 \%$, values previously used to claim success [53]. The ProMate's authors reported a success rate for protein-protein binding site prediction of $70 \%$ [55], while application of the criteria used in PPI-PRED gave ProMate's success rate as 36\% [53]. According to our data [see Additional file 2], the prediction with an AUC $\geq 0.6$ corresponded to a significant prediction (P-value $<0.05)$ at a sensitivity $>30 \%$. Using an AUC $\geq 0.6$ as a criterion of successful prediction, PPI-PRED gave $60 \%$ and ProMate $35 \%$ successful predictions, respectively (Fig. 10). Neither ProMate nor PPI-PRED used antibody-protein interfaces for their methods development; nevertheless, they predicted epitopes with a success rate comparable to those for prediction of protein interfaces.

Epitopes and other protein-protein interfaces indeed share many properties. Thus, Blythe [62] compared 57 protein-protein binding interfaces of 44 proteins from the dataset used for ProMate development [55] with epitopes and paratopes inferred from X-ray structures of $37 \mathrm{com}-$

Table 4: Overall performance of patch prediction and protein-protein docking methods

\begin{tabular}{|c|c|c|c|c|c|c|c|c|c|}
\hline statistics & ProMate & $\begin{array}{l}\text { PPI-PRED } \\
\text { | st patch }\end{array}$ & $\begin{array}{l}\text { PPI-PRED } \\
\text { best patch }\end{array}$ & $\begin{array}{l}\text { PatchDock } \\
\text { | st model }\end{array}$ & $\begin{array}{l}\text { PatchDock best } \\
\text { model of } 10\end{array}$ & $\begin{array}{c}\text { ClusPro (DOT) } \\
\text { I st model }^{\text {st }}\end{array}$ & $\begin{array}{l}\text { ClusPro (DOT) } \\
\text { best model of } 10\end{array}$ & CEP & $\begin{array}{l}\text { DiscoTope } \\
(-7.7)\end{array}$ \\
\hline sensitivity & 0.091 & 0.153 & 0.331 & 0.300 & 0.425 & 0.258 & 0.453 & 0.310 & 0.416 \\
\hline I-specificity & 0.083 & 0.161 & 0.135 & 0.135 & 0.114 & 0.079 & 0.067 & 0.223 & 0.214 \\
\hline PPV & 0.101 & 0.083 & 0.188 & 0.175 & 0.262 & 0.235 & 0.390 & 0.110 & 0.155 \\
\hline accuracy & 0.841 & 0.780 & 0.819 & 0.816 & 0.846 & 0.863 & 0.892 & 0.739 & 0.754 \\
\hline AUC & 0.504 & 0.496 & 0.598 & 0.583 & 0.656 & 0.589 & 0.693 & 0.544 & 0.601 \\
\hline P-value & 0.27 & 1.0 & $7.8 \mathrm{E}-30$ & $9.0 \mathrm{E}-23$ & $<1.0 \mathrm{E}-50$ & 7.9E-34 & $<1.0 \mathrm{E}-50$ & $4.3 \mathrm{E}-06$ & 4.IE-25 \\
\hline \multicolumn{10}{|c|}{ Statistics averaged over epitopes } \\
\hline sensitivity & $0.09 \pm 0.17$ & $0.15 \pm 0.24$ & $0.34 \pm 0.32$ & $0.27 \pm 0.24$ & $0.42 \pm 0.29$ & $0.25 \pm 0.31$ & $0.46 \pm 0.28$ & $0.34 \pm 0.28$ & $0.43 \pm 0.31$ \\
\hline I-specificity & $0.08 \pm 0.03$ & $0.16 \pm 0.07$ & $0.14 \pm 0.07$ & $0.15 \pm 0.06$ & $0.13 \pm 0.07$ & $0.10 \pm 0.07$ & $0.08 \pm 0.05$ & $0.28 \pm 0.20$ & $0.22 \pm 0.15$ \\
\hline PPV & $0.11 \pm 0.20$ & $0.10 \pm 0.17$ & $0.21 \pm 0.24$ & $0.18 \pm 0.19$ & $0.30 \pm 0.25$ & $0.25 \pm 0.33$ & $0.41 \pm 0.29$ & $0.11 \pm 0.08$ & $0.18 \pm 0.12$ \\
\hline accuracy & $0.83 \pm 0.05$ & $0.77 \pm 0.07$ & $0.81 \pm 0.08$ & $0.80 \pm 0.08$ & $0.83 \pm 0.09$ & $0.84 \pm 0.09$ & $0.88 \pm 0.07$ & $0.69 \pm 0.17$ & $0.74 \pm 0.12$ \\
\hline AUC & $0.51 \pm 0.09$ & $0.50 \pm 0.13$ & $0.60 \pm 0.17$ & $0.56 \pm 0.11$ & $0.64 \pm 0.17$ & $0.58 \pm 0.17$ & $0.69 \pm 0.15$ & $0.53 \pm 0.08$ & $0.60 \pm 0.13$ \\
\hline
\end{tabular}




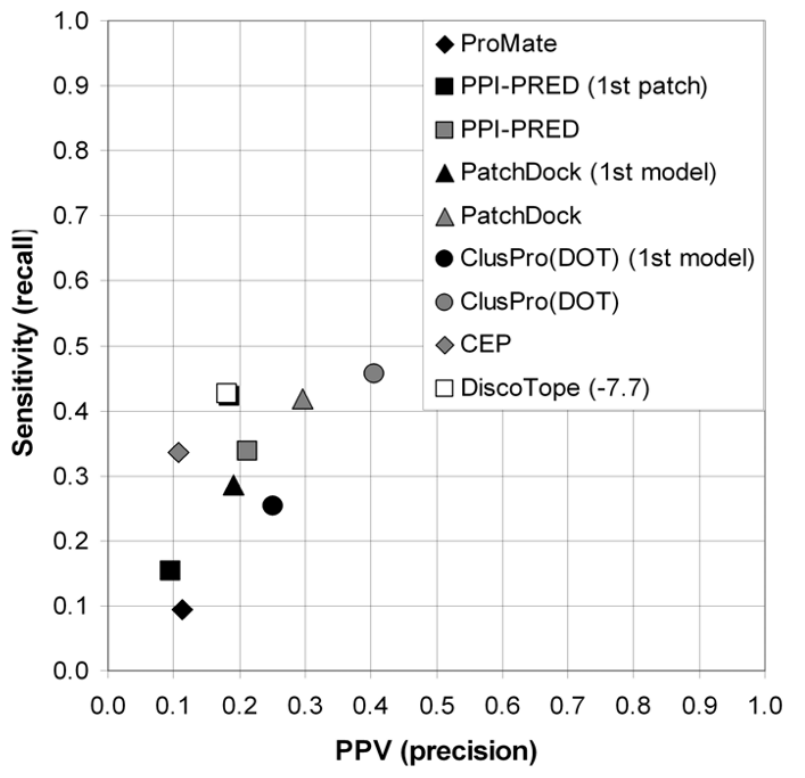

Figure 9

Overall methods performance measured as average sensitivity and PPV values. plexes calculating the following interface properties: amino acid composition, hydrophobicity by the Eisenberg's scale [63], amino acid contribution to form intermolecular hydrogen bonds, residue evolutionary conservancy, and several geometrical parameters, such as planarity and complementarity of interfaces. Epitopes and non-obligate heterodimer interfaces were very similar considering all the aforementioned properties except residue conservancy; epitope residues were more variable than heterodimer interfaces [62]. The current work additionally demonstrates that, on average, epitope residues are significantly less conservative than protein surface residues. Indeed, protein-protein interaction sites are under evolutionary pressure to be more conserved than protein surface residues on average. While antibody-antigen interactions are not under evolutionary pressure, they are under the selection pressure from the host immune system. This selection pressure is assumed to cause polymorphisms in pathogens and to explain the variability of immune epitopes.

\section{Conclusion}

Benchmark datasets for use in B cell structural epitope prediction have been constructed and made available. Using these benchmark data, eight publicly available web serv-

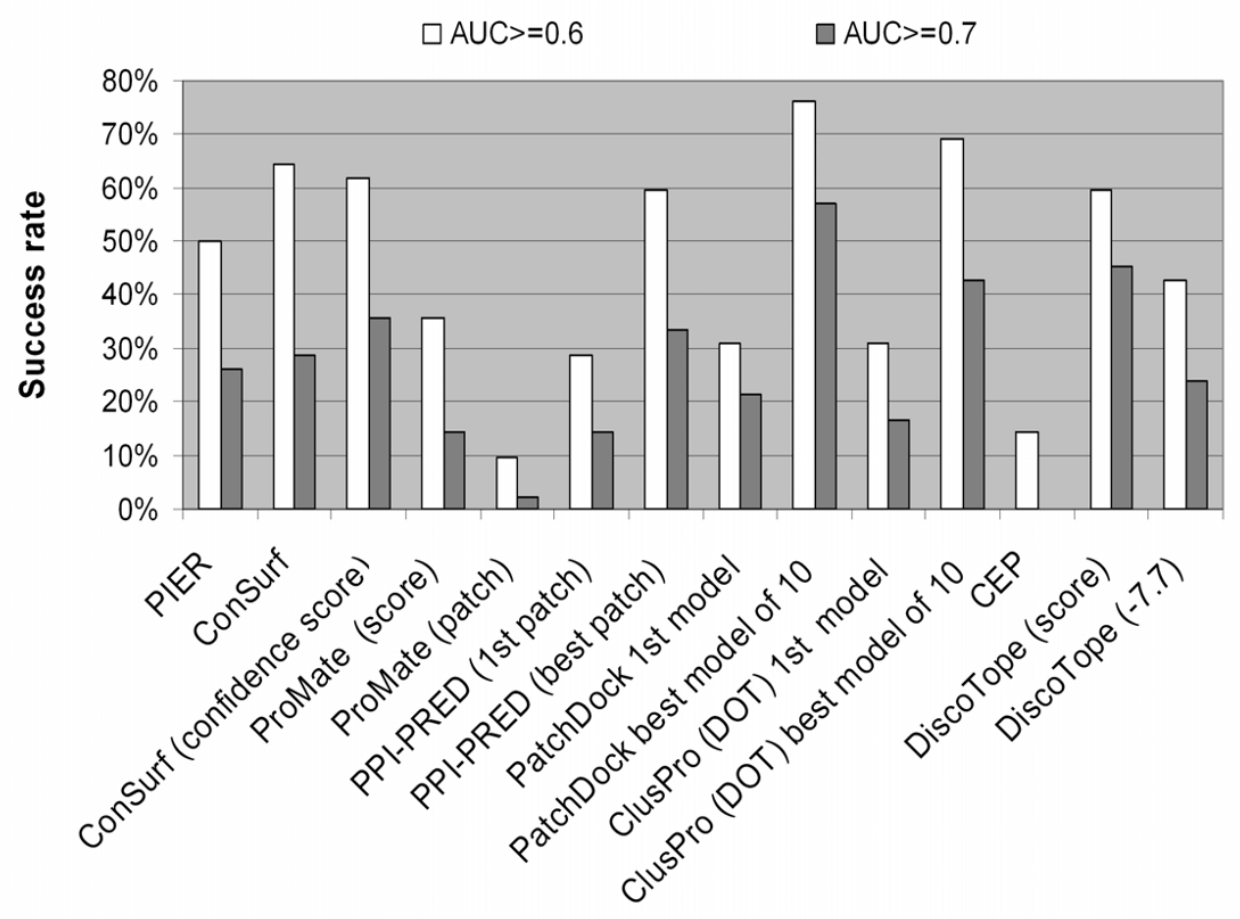

Figure 10

Proportion of successfully predicted epitopes. 
ers and their associated methods were evaluated. Several schemes for methods evaluation were considered.

The overall performance was poor for all methods and did not exceed an average AUC of 0.7 and $40 \%$ positive predictive value (precision) at $46 \%$ sensitivity (recall). The values of the area under the receiver operating characteristic (ROC) curve for the evaluated methods were about 0.6 for ConSurf, DiscoTope, and PPI-PRED (when all predictions were considered) and above 0.65 but not exceeding 0.7 for protein-protein docking methods when the best of the top ten models for the bound docking were considered. Certainly a best case, since under real conditions many more models would be presented. Other methods, PIER, ProMate (both scores and patch prediction), CEP, PPI-PRED first patch, and the first models of docking methods, performed close to random. Despite the fact that structural epitopes and protein-protein non-obligate transient heterodimer interfaces share many properties, protein-protein binding site prediction methods were poor epitope predictors.

When the top ten models and bound docking were considered, the docking methods performed the best, especially PatchDock, where success can be explained by application of the CDR filter, which the DOT algorithm does not use. Independent evaluation of PPI-PRED and four docking algorithms (DOT, PatchDock, ZDOCK, and webGRAMM) made by Martin Blythe [62] and not available to us until the peer-reviewing stage of the manuscript agrees with the results presenting in the current work. Using the Matthew's Correlation Coefficient (MCC), Blythe measured the correlation between predicted and structural epitopes and paratopes inferred from 37 antibody-protein complexes. For the first models, all evaluated methods demonstrated near random correlations. Likewise, when the top ten models for each complex were considered, low and negative MCC values prevailed over positive values for all algorithms except PatchDock. Further experiments demonstrated that using the CDR filter may improve the prediction. Thus, using predefined CDRs for antibodies, the DOT method significantly improved and showed MCC values comparable with those for PatchDock [62].

Obviously, unbound docking would have more practical value for epitope prediction than bound docking. However, the performance of unbound docking for antibodyantigen interactions, as was shown by the authors of PatchDock, was unsatisfactory in comparison to bound docking and other protein-protein interaction methods [64]. While the bound docking considered in this work has no practical value for epitope prediction, it needs to be benchmarked to further improve unbound docking algorithms and tune them for modeling antibody-antigen complexes.

Currently the problem of B-cell epitope prediction is far from solved: structure-based method for prediction of discontinuous epitopes perform on the same level as sequence-based methods for prediction of continuous epitopes giving the area under the receiver operating characteristic curve (AUC) values of approximately 0.60 [25].

Three definitions of an epitope inferred from the X-ray structure of antibody-protein complexes were considered, but this made no significant difference to the predictions. Hence, we finally considered an epitope residue as the protein antigen residue for which any atom is separated from any antibody atom by a distance $\leq 4 \AA$.

Currently, each method requires writing a separate parser taking into account different representations of the output data. There is a need to develop a common format for output data generated by both scale-based and patch generation tools that is easily interpreted by both a human and computer.

Given these shortcomings and current success rates, how can epitope prediction be improved? The availability of larger datasets containing only well-defined epitopes inferred from X-ray structures of antibody-protein complexes, which are then used for training, would help. This will come over time as the PDB continues to grow at a rapid rate. This need, in the context of continuous epitope prediction, has been noted by others [25]. The performance of docking algorithms might be improved by tuning them specifically for antibody-antigen complexes. Existing B-cell epitope prediction methods utilize only a few features characterizing epitopes (amino acid propensities, residue solvent accessibility, spatial distribution, and inter-molecular contacts). Therefore, another possible way for improving the prediction would be to utilize more features that discriminate epitopes from non-epitopes, for example, the evolutionary conservation score. This assumes that an epitope is indeed a discreet entity based upon what we know about proteins today. Perhaps the more fundamental question is whether it makes sense to consider a B-cell epitope a discrete feature of a protein at all? Time will tell as more X-ray structures on antibodyprotein complexes become available.

\section{Methods \\ Surface residue}

is defined as a protein residue with a relative ASA of $\geq 1 \%$ as calculated by the program NACCESS. This cut-off was previously used by Jones \& Thornton [46]. 


\section{Data sets compilation}

169 structures of protein antigens (length $>30$ amino acids) in complex with antibody fragments have been manually collected from the PDB [41] of January 2006 at a resolution $\leq 4 \AA$. Every structure has been manually curated within the IEDB database [1] and inspected using the EpitopeViewer visualization tool developed by the authors [65]. Structures in which the antibody binds antigen but involves no CDR residues have been excluded from the analysis; there were four such structures [PDB: $1 \mathrm{MHH}, 1 \mathrm{HEZ}, 1 \mathrm{DEE}, 1 \mathrm{IGC}$. If a structure contained several complexes in one asymmetric unit (there were 46 such structures in 165) and the authors of the structure observed no structural difference between these complexes, only one complex was selected - those that were specified as a reference complex by the authors of the article describing the structure (primary citation in the PDB); there were 18 such structures out of 46 . If the authors didn't provide this information, all complexes in the structure were considered for analysis. The authors of a few structures clearly stated in their papers that antibodyprotein contacts in the complexes were different: [PDB: 1MLC, 1NFD, 1OB1, 1P2C, 1QFW]. This initial curation has performed in order to correctly assign the proteinantibody complexes and decrease the number of individual complexes analyzed from 226 to 187 from a total of 169 structures. A total of 24 complexes were formed by one-chain antibody fragments and 163 complexes by twochain antibody fragments. Alignment of protein chains was performed using the CE algorithm [58].

\section{Web-servers evaluation}

The publicly available web-servers implementing 3D structure-based methods for protein-protein binding site and/or discontinuous epitope prediction were identified through PubMed and web searches. Eight web-servers were selected for evaluation (Table 1). The servers were tested between June and September of 2006, and results reflect the method implemented by the servers at that time. In all cases the default parameters provided by each server were used.

PPI-PRED provides up to three surface patches predicted as putative binding sites. The batch mode for data submission was used.

CEP provides residues forming the putative conformational epitopes (there could be more than 20 predictions per protein antigen). CEP includes residues with accessibility less than $25 \%$. In this work, only residues with accessibility more than $25 \%$ were considered as a part of the epitope.

DiscoTope assigns a score to each protein residue that reflects the probability of that residue being part of an epitope and also provides a list of residues included in the predicted epitope (patch). DiscoTope predicts one epitope per protein.

ProMate returns results in four different formats. In this work, the two formats provided for each residue patch/ non-patch identifier and residue interface probability were used. The batch version of ProMate, MultiProMate, was used.

PIER returns a list of residues with assigned PIER index values indicating how likely a particular residue is to be involved in protein interface formation, with higher values meaning higher probability. A PIER index above 30 indicates a likely protein-protein binding interface residue, and below zero an unlikely interface residue.

ConSurf calculates a conservation score for each protein residue based on a PSI-BLAST alignment of unique homologous sequences found in UniProtKB/Swiss-Prot [66]. For each protein residue, ConSurf provides a normalized score, so that the average score for all residues in the protein is zero, and the standard deviation is one. The conservation scores calculated by ConSurf are a relative measure of the evolutionary conservation at each residue of the target protein. The lowest scores represent the most conserved positions in the protein. ConSurf provides output data in different formats. In this analysis the "Amino Acid Conservation Score" output files were used. These files provide, together with normalized conservation score for each residue, residue color values (scale of 1-9) and confidence intervals for the conservation score and color (for the Bayesian method of calculation which is used by default). Amino acid positions that are assigned confidence intervals that are too large to be trustworthy are marked in the output files. Both all residues with conservation scores and residues for which scores were confident (not marked in the output files of the ConSurf server), i.e., a confident interval assigned to the score was less than $50 \%$ [56], were used in this study.

ClusPro running the DOT program returns the ten best models as one PDB formatted file re-numerating protein chains, residues and atoms. DOT is limited to proteins not exceeding 3,700 atoms.

PatchDock returns up to 2,000 models each as a separate PDB formatted file and provides the option to retain the 100 best models in one archive file. The ten best (by model score) were used in the current analysis. Also the filter for antigen-antibody interactions provided by PatchDock was used. That is, surface patches intersecting the CDR regions of the antibody. CDRs are detected by aligning the sequence of the given antibody to a consensus sequence from a library of antibodies [64]. 
ClusPro and PatchDock differ from the other servers tested by providing protein-protein docking. To use these servers the user needs to provide the structure of the antibody along with the antigen structure. We used the structures of protein antigen and antibody from the same complex, hence, only bound docking was considered. As was shown by the authors of the method, PatchDock bound docking substantially out-performed unbound docking [64].

The AUC values for scale-based methods were calculated using the algorithm of Tom Fawcett [61]. For discrete classifiers, that is, methods producing the only point on the ROC plot with coordinates $\{x ; y\}$, the AUC was calculating as $0.5^{*}(1-x+y)$.

Molecule images were produced using the WebLabViewer software (Accelrys Inc.).

\section{Abbreviations}

CDR - Complementary Determined Region of the Antibody.

Fab - antigen-binding fragment of antibody that includes one complete light chain paired with one heavy chain fragment containing the variable domain and the first constant domain.

VHH - antigen-binding fragment of the antibody that includes the variable domain of the heavy chain.

$\mathrm{Fv}$ - antigen-binding fragment of antibody that includes variable domains of heavy and light chains.

$\mathrm{scFv}$ - antigen-binding fragment of the antibody that includes the covalently linked variable domains of the heavy and light chains.

TCR - T Cell receptor.

$\overline{\mathrm{x}}$ - sample arithmetic mean.

$s$ - sample standard deviation.

TP, FP, TN, FN - true positives, false positives, true negatives, and false negatives, respectively.

ROC - Receiver Operating Characteristics.

AUC - area under the ROC curve.

\section{Authors' contributions}

JVP conceived, designed and performed the research including data collection and analysis. PEB suggested extensions and modifications to the research. Both JVP and PEB wrote the manuscript. The authors have read and approved the final version of the manuscript.

\section{Additional material}

\section{Additional file 1}

The representative structures of protein antigens (numbered) and antibody-protein complexes represented different epitopes for each antigen (epitopes inferred from one-chain antibody fragments are in italic). The data provides curated information on $823 D$ structures of antibody-protein complexes (dataset \#1) represented 169 structures of antibody-protein complexes available in the PDB of January, 2006 and used in this work.

Click here for file

[http://www.biomedcentral.com/content/supplementary/14726807-7-64-S1.doc]

\section{Additional file 2}

The detailed statistics on the prediction results for 59 representative epitope. This table provides additional information that complements the Tables 2 and 3. The analysis was performed using 59 representative epitopes from dataset \#2 that were inferred from structures of one-chain (monomer) antigens in complexes with two-chain antibody fragments. Click here for file

[http://www.biomedcentral.com/content/supplementary/14726807-7-64-S2.xls]

\section{Acknowledgements}

The work was supported by the National Institutes of Health Contract HHSN26620040006C. The authors would like to acknowledge Dr. Alex Sette and Dr. Bjoern Peters from the La Jolla Institute of Allergy and Immunology (LIAI) and Dr. Ole Lund from the Technical University of Denmark for helpful discussions.

\section{References}

I. Peters B, Sidney J, Bourne P, Bui HH, Buus S, Doh G, Fleri W, Kronenberg $M$, Kubo R, Lund O, Nemazee D, Ponomarenko JV, Sathiamurthy M, Schoenberger SP, Stewart S, Surko P, Way S, Wilson S, Sette $A$ : The design and implementation of the immune epitope database and analysis resource. Immunogenetics 2005 , 57(5):326-336.

2. Van Regenmortel MH: Immunoinformatics may lead to a reappraisal of the nature of $B$ cell epitopes and of the feasibility of synthetic peptide vaccines. I Mol Recognit 2006, 19(3): $183-187$.

3. Gomara MJ, Haro I: Synthetic peptides for the immunodiagnosis of human diseases. Curr Med Chem 2007, I 4(5):53I-546.

4. Meloen RH, Puijk WC, Langeveld JP, Langedijk JP, Timmerman P: Design of synthetic peptides for diagnostics. Curr Protein Pept Sci 2003, 4(4):253-260.

5. Leinikki $P$, Lehtinen $M$, Hyoty $H$, Parkkonen $P$, Kantanen $M L$, Hakulinen J: Synthetic peptides as diagnostic tools in virology. Adv Virus Res 1993, 42:149-186.

6. Eigenmann PA: Do we have suitable in-vitro diagnostic tests for the diagnosis of food allergy? Curr Opin Allergy Clin Immunol 2004, 4(3): $211-2 \mid 3$

7. Routsias JG, Vlachoyiannopoulos PG, Tzioufas AG: Autoantibodies to intracellular autoantigens and their B-cell epitopes: molecular probes to study the autoimmune response. Crit Rev Clin Lab Sci 2006, 43(3):203-248.

8. Arnon R: Synthetic Vaccines. Boca Raton, FL, CRC Press; 1987.

9. Arnon $\mathrm{R}$, Ben-Yedidia $\mathrm{T}$ : Old and new vaccine approaches. Int Immunopharmacol 2003, 3(8): I I95-I 204. 
10. Hans D, Young PR, Fairlie DP: Current status of short synthetic peptides as vaccines. Med Chem 2006, 2(6):627-646.

II. Davies MN, Flower DR: Harnessing bioinformatics to discover new vaccines. Drug Discov Today 2007, I 2(9-1 0):389-395.

12. Taylor PD, Flower DR: Immunoinformatics and Computationa Vaccinology: A Brief Introduction. In In Silico Immunology Edited by: Flower DR, Timmis J. Springer; 2007:23-46.

13. Hopp TP, Woods KR: Prediction of Protein Antigenic Determinants from Amino Acid Sequences. Proc Natl Acad Sci U S A 1981, 78:3824-3828.

14. Emini EA, Hughes JV, Perlow DS, Boger J: Induction of hepatitis A virus-neutralizing antibody by a virus-specific synthetic peptide. J Virol 1985, 55(3):836-839.

15. Garnier J, Osguthorpe DJ, Robson B: Analysis of the accuracy and implications of simple methods for predicting the secondary structure of globular proteins. J Mol Biol 1978, I 20(1):97-I 20.

16. Pellequer JL, Westhof E, Van Regenmortel MH: Correlation between the location of antigenic sites and the prediction of turns in proteins. Immunol Lett 1993, 36(I):83-99.

17. Levitt M: Conformational preferences of amino acids in globular proteins. Biochemistry 1978, I7(20):4277-4285.

18. Chou PY, Fasman GD: Conformational parameters for amino acids in helical, beta-sheet, and random coil regions calculated from proteins. Biochemistry 1974, 13(2):21 I-222.

19. Larsen JE, Lund O, Nielsen M: Improved method for predicting linear B-cell epitopes. Immunome Res 2006, 2:2.

20. Saha S, Raghava GP: Prediction of continuous B-cell epitopes in an antigen using recurrent neural network. Proteins 2006 , 65(I):40-48

21. Chen R, Li L, Weng Z: ZDOCK: an initial-stage protein-docking algorithm. Proteins 2003, 52(I):80-87.

22. Sollner J, Mayer B: Machine learning approaches for prediction of linear B-cell epitopes on proteins. J Mol Recognit 2006 19(3):200-208.

23. Sollner J: Selection and combination of machine learning classifiers for prediction of linear B-cell epitopes on proteins. Mol Recognit 2006, 19(3):209-2।4.

24. Blythe MJ, Flower DR: Benchmarking B cell epitope prediction: underperformance of existing methods. Protein Sci 2005 , I 4(I):246-248.

25. Greenbaum JA, Andersen PH, Blythe M, Bui HH, Cachau RE, Crowe J, Davies M, Kolaskar AS, Lund O, Morrison S, Mumey B, Ofran Y, Pellequer JL, Pinilla C, Ponomarenko JV, Raghava GP, van Regenmortel MH, Roggen EL, Sette A, Schlessinger A, Sollner J, Zand M, Peters $B$ : Towards a consensus on datasets and evaluation metrics for developing B-cell epitope prediction tools. J Mol Recognit 2007, 20(2):75-82.

26. Westhof E, Altschuh D, Moras D, Bloomer AC, Mondragon A, Klug $A$, Van Regenmortel $\mathrm{MH}$ : Correlation between segmental mobility and the location of antigenic determinants in proteins. Nature 1984, 3 I I(5982): 123- | 26

27. Tainer JA, Getzoff ED, Alexander H, Houghten RA, Olson AJ, Lerner RA, Hendrickson WA: The reactivity of anti-peptide antibodies is a function of the atomic mobility of sites in a protein Nature 1984, 3 I 2(5990): I 27- I34.

28. Novotny J. Handschumacher M, Haber E, Bruccoleri RE, Carlson WB, Fanning DW, Smith JA, Rose GD: Antigenic determinants in proteins coincide with surface regions accessible to large probes (antibody domains). Proc Natl Acad Sci U S A 1986, 83(2):226-230.

29. Thornton JM, Edwards MS, Taylor WR, Barlow DJ: Location of 'continuous' antigenic determinants in the protruding regions of proteins. Embo J 1986, 5(2):409-4I3.

30. Amit AG, Mariuzza RA, Phillips SE, Poljak RJ: Three-dimensional structure of an antigen-antibody complex at $2.8 \mathrm{~A}$ resolution. Science 1986, 233(4765):747-753.

31. Kolaskar AS, Kulkarni-Kale U: Prediction of three-dimensional structure and mapping of conformational epitopes of envelope glycoprotein of Japanese encephalitis virus. Virology 1999, 26I(I):3I-42

32. Laver WG, Air GM, Webster RG, Smith-Gill SJ: Epitopes on protein antigens: misconceptions and realities. Cell 1990, 6I(4):553-556.

33. Van Regenmortel MHV: Mapping Epitope Structure and Activity: From One-Dimensional Prediction to Four-Dimensional Description of Antigenic Specificity. Methods 1996 9(3):465-472.
34. Bajaj K, Chakrabarti P, Varadarajan R: Mutagenesis-based definitions and probes of residue burial in proteins. Proc Natl Acad Sci U S A 2005, 102(45): 16221-16226.

35. Van Regenmortel MH: Structural and functional approaches to the study of protein antigenicity. Immunol Today 1989, I0(8):266-272.

36. Benjamin DC, Perdue SS: Site-Directed Mutagenesis in Epitope Mapping. Methods 1996, 9(3):508-5I5.

37. Cunningham BC, Wells JA: Comparison of a structural and a functional epitope. J Mol Biol I993, 234(3):554-563.

38. Novotny J, Bruccoleri RE, Saul FA: On the attribution of binding energy in antigen-antibody complexes McPC 603, DI.3, and HyHEL-5. Biochemistry 1989, 28(I I ):4735-4749.

39. Novotny J: Protein antigenicity: a thermodynamic approach. Mol Immunol I99|, 28(3):20|-207.

40. Lo Conte L, Chothia C, Janin J: The atomic structure of proteinprotein recognition sites. J Mol Biol I999, 285(5):2I77-2। 98 .

4I. Berman HM, Westbrook J, Feng Z, Gilliland G, Bhat TN, Weissig H, Shindyalov IN, Bourne PE: The Protein Data Bank. Nucleic Acids Res 2000, 28(I):235-242.

42. Jones $S$, Thornton JM: Principles of protein-protein interactions. Proc Natl Acad Sci U S A 1996, 93(I):13-20.

43. Nooren IM, Thornton IM: Diversity of protein-protein interactions. Embo J 2003, 22(1 4):3486-3492.

44. Kleanthous C: Protein-Protein Recognition. In Fronties in Molecular Biology New York, Oxford University press; 2000.

45. Janin J: Kinetics and thermodynamics of protein-protein interactions. In Protein-Protein Recognition Edited by: Kleanthous C. New York, Oxford University Press; 2000:I-32.

46. Jones S, Thornton JM: Prediction of protein-protein interaction sites using patch analysis. J Mol Biol I997, 272(I): I33-I 43.

47. Jones $\mathrm{S}$ Thornton, J.: Analysis and classification of protein-protein interactions from a structural perspective. In Protein-Protein Recognition Edited by: Kleanthous C. New York , Oxford University Press; 2000:33-59.

48. Kulkarni-Kale U, Bhosle S, Kolaskar AS: CEP: a conformational epitope prediction server. Nucleic Acids Res 2005, 33(Web Server issue): WI68-7I.

49. Haste Andersen P, Nielsen M, Lund O: Prediction of residues in discontinuous B-cell epitopes using protein 3D structures. Protein Sci 2006, I5(I I):2558-2567.

50. Comeau SR, Gatchell DW, Vajda S, Camacho CJ: ClusPro: an automated docking and discrimination method for the prediction of protein complexes. Bioinformatics 2004, 20(I):45-50.

5I. Mandell JG, Roberts VA, Pique ME, Kotlovyi V, Mitchell JC, Nelson E, Tsigelny I, Ten Eyck LF: Protein docking using continuum electrostatics and geometric fit. Protein Eng 200 I, I 4(2): I05-I I3.

52. Schneidman-Duhovny D, Inbar Y, Polak V, Shatsky M, Halperin I, Benyamini $H$, Barzilai $A$, Dror $O$, Haspel N, Nussinov R, Wolfson $H J$ : Taking geometry to its edge: fast unbound rigid (and hingebent) docking. Proteins 2003, 52(I): I07-I I 2.

53. Bradford JR, Westhead DR: Improved prediction of protein-protein binding sites using a support vector machines approach. Bioinformatics 2005, 21: | 487-1494.

54. Kufareva I, Budagyan L, Raush E, Totrov M, Abagyan R: PIER: protein interface recognition for structural proteomics. Proteins 2007, 67(2):400-417.

55. Neuvirth H, Raz R, Schreiber G: ProMate: a structure based prediction program to identify the location of protein-protein binding sites. J Mol Biol 2004, 338(I): I8I-199.

56. Landau M, Mayrose I, Rosenberg Y, Glaser F, Martz E, Pupko T, BenTal N: ConSurf 2005: the projection of evolutionary conservation scores of residues on protein structures. Nucleic Acids Res 2005, 33(Web Server issue):W299-302.

57. Hubbard SJ: NACCESS Computer Program. University College London; 1993.

58. Shindyalov IN, Bourne PE: Protein structure alignment by incremental combinatorial extension (CE) of the optimal path. Protein Eng 1998, II(9):739-747.

59. Fleury D, Daniels RS, Skehel J], Knossow M, Bizebard T: Structural evidence for recognition of a single epitope by two distinct antibodies. Proteins 2000, 40(4):572-578.

60. Huang CC, Venturi M, Majeed S, Moore MJ, Phogat S, Zhang MY, Dimitrov DS, Hendrickson WA, Robinson J, Sodroski J, Wyatt R, Choe H, Farzan M, Kwong PD: Structural basis of tyrosine sulfation and $\mathrm{VH}$-gene usage in antibodies that recognize the HIV 
type I coreceptor-binding site on gp I 20. Proc Natl Acad Sci U S A 2004, I0I(9):2706-27II.

61. Fawcett T: An introduction to ROC analysis. In Pattern Recognition Letters Volume 27. Elsevier; 2006:86I-874.

62. Blythe MJ: Computational Characterisation of B cell Epitopes. In School of Animal and Microbial Sciences, The Edward Jenner Institute for Vaccine Research Volume Ph.D.. The University of Reading; 2006:243.

63. Eisenberg D, Weiss RM, Terwilliger TC: The hydrophobic moment detects periodicity in protein hydrophobicity. Proc Natl Acad Sci U S A 1984, 8 I (1): I40-I 44

64. Duhovny D Nussinov, R, Wolfson, HJ.: Efficient Unbound Docking of Rigid Molecules. In 2'nd Workshop on Algorithms in Bioinformatics(WABI) Rome, Italy, Lecture Notes in Computer Science 2452 Edited by: D. GRG. Springer Verlag; 2002:185-200.

65. Beaver JE, Bourne PE, Ponomarenko JV: EpitopeViewer: a Java application for the visualization and analysis of immune epitopes in the Immune Epitope Database and Analysis Resource (IEDB). Immunome Res 2007, 3:3.

66. The Universal Protein Resource (UniProt). Nucleic Acids Res 2007, 35(Database issue):DI93-7.

Publish with Bio Med Central and every scientist can read your work free of charge

"BioMed Central will be the most significant development for disseminating the results of biomedical research in our lifetime. "

Sir Paul Nurse, Cancer Research UK

Your research papers will be:

- available free of charge to the entire biomedical community

- peer reviewed and published immediately upon acceptance

- cited in PubMed and archived on PubMed Central

- yours - you keep the copyright

Submit your manuscript here:

http://www.biomedcentral.com/info/publishing_adv.asp
BiolMedcentral 